\title{
Taste-split staggered actions: eigenvalues, chiralities and Symanzik improvement
}

\author{
Stephan Dürr ${ }^{a, b}$ \\ ${ }^{a}$ Bergische Universität Wuppertal, Gaußstraße 20, 42119 Wuppertal, Germany \\ bJülich Supercomputing Center, Forschungszentrum Jülich, 52425 Jülich, Germany
}

\begin{abstract}
The eigenvalue spectra of staggered fermions with an Adams and/or Hoelbling mass term are studied. The chiralities of the eigenmodes reflect whether the chirality linked to the unflavored approximate $\left(\gamma_{5} \times 1\right)$ or the flavored exact $\left(\gamma_{5} \times \xi_{5}\right)$ staggered symmetry is considered, and which one of the RR, LR, RL, LL eigenmode definitions is used. In either case a sensitivity to the topological charge of the gauge background is found. We discuss how to remove the leading cut-off effects of these actions by means of a properly tuned improvement term and/or the overlap procedure. The combination of Symanzik improvement and link smearing radically improves the properties of the physical branch.
\end{abstract}

\section{Introduction}

The so-called fermion doubling problem in lattice field theory has been addressed in many ways; the two most popular approaches are known as Wilson fermions [1] and staggered fermions [2], respectively. A problem being cured in several ways usually indicates that none of the solutions is completely satisfactory in all respects, and the lattice is no exception to this rule.

Wilson fermions represent the spinor components as internal degrees of freedom (i.e. with explicit $\gamma$-matrices), such that on a lattice with $N$ grid points the Wilson formulation entails a matrix of size $4 N_{c} N \times 4 N_{c} N$, where $N_{c}$ is the number of colors. Susskind "staggered" fermions are based on the observation that the eigenvalue spectra of naive fermions on interacting backgrounds are 4-fold degenerate (i.e. not 16-fold in 4 space-time dimensions as one might have naively guessed), and hence a reduction to 1-component spinors is possible (at the price of distributing the spinor degrees of freedom over space-time), such that one ends up with a staggered fermion matrix of size $N_{c} N \times N_{c} N$ that corresponds to 4 species in the continuum.

Wilson fermions are convenient for their conceptual simplicity (there is a 1-to-1 correspondence between lattice and continuum flavor). Their main technical disadvantage is the breaking of chiral symmetry through the dimension 5 operator $\bar{\psi}(-\triangle) \psi$ that is added to lift 15 out of the 16 naive species to a mass of order $1 / a$, where $\triangle$ is the gauge covariant Laplacian and $a$ is the lattice spacing. Staggered fermions offer the advantage of a truncated form of chiral symmetry and of more speedy simulations (the size of the matrix is smaller). Their main technical disadvantage is that the taste symmetry (among the 4 species) is not exact and that the reduction to a single species proceeds in different ways for sea-quarks (those which come from the functional determinant) and valence-quarks (those which stem from interpolating fields). This renders the rooted staggered formulation (at finite $a$ ) non-local and/or non-unitary which, 
in principle, could affect the universality class of the theory (though there is plenty of analytical and numerical evidence that this does not happen), see e.g. the review [3].

In either approach the main technical disadvantage can be mitigated. Replacing the gauge links $U_{\mu}(x)$ of the covariant derivative $\left(\nabla_{\mu} \phi\right)(x)=\left[U_{\mu}(x) \phi(x+\hat{\mu})-U_{\mu}^{\dagger}(x-\hat{\mu}) \phi(x-\hat{\mu})\right] / 2$ in the operator by smeared links $V_{\mu}(x)$ (see below for details) reduces the amount of chiral symmetry breaking with Wilson fermions (particularly effectively when combined with a clover term) and of taste symmetry breaking with staggered fermions (see $[3]$ for a guide to the literature). In the Wilson case chiral symmetry breaking can be completely removed through the overlap procedure [4,5]; unfortunately this increases the computational requirements by a factor $O(100)$.

Recently, two modifications of the staggered action $S_{\mathrm{S}}=\bar{\chi}\left[D_{\mathrm{S}}+m\right] \chi$ with $D_{\mathrm{S}}=\eta_{\mu} \nabla_{\mu}$ and $\eta_{\mu}(x)$ given below were proposed which go by the somewhat confusing name "staggered Wilson fermions". In this article we call them "taste-split staggered actions". In essence the proposal is to replace/augment the usual staggered mass term $m(1 \otimes 1)$ in $S_{\mathrm{S}}$, where the notation is spinor $\otimes$ taste $[6]$, by taste non-singlet mass terms $\propto(1 \otimes \xi)$ which are designed to (partly or fully) lift the staggered near-degeneracy, i.e. some species get masses $2 / a$ or $4 / a$ such that they decouple in the continuum limit. Such non-standard staggered mass terms were first considered in [8] where it was noticed that they break the remnant chiral symmetry $D_{\mathrm{S}}(x, y) \rightarrow e^{\mathrm{i} \epsilon(x) \theta} D_{\mathrm{S}}(x, y) e^{\mathrm{i} \epsilon(y) \theta}$ of the massless staggered action [with $\epsilon(x)$ defined below] more severely than the usual mass term does. The Adams proposal [9]

$$
S_{\mathrm{A}}=\bar{\chi}\left[\eta_{\mu} \nabla_{\mu}+r\left(M_{\mathrm{A}}+1\right)\right] \chi
$$

builds on a mass term $M_{\mathrm{A}} \simeq 1 \otimes \xi_{5}$, such that in the naive continuum limit two species stay massless, while two have mass $2 r / a$ (where $0<r<2$ is akin to the Wilson parameter); the precise form of $M_{\mathrm{A}}$ will be given below. Similarly, Hoelbling proposes the two operators [10]

$$
\begin{gathered}
S_{\text {Hori }}=\bar{\chi}\left[\eta_{\mu} \nabla_{\mu}+r\left(M_{\mathrm{A}}+M_{\mathrm{Hori}}+2\right)\right] \chi \\
S_{\mathrm{Hsym}}=\bar{\chi}\left[\eta_{\mu} \nabla_{\mu}+r\left(M_{\mathrm{Hsym}}+2\right)\right] \chi
\end{gathered}
$$

which fully lift the staggered near-degeneracy, i.e. one species stays massless, while two receive a mass $2 r / a$ and the last one is brought up to $4 r / a$. The two versions differ on how they break the rotational symmetry group $R_{\mu \nu}$ of the massless staggered action; the precise form of $M_{\text {Hori }}$ and $M_{\text {Hsym }}$ will be given below. It is straight forward to write down the linear combination

$$
S_{\mathrm{Hmix}}=\bar{\chi}\left[\eta_{\mu} \nabla_{\mu}+\frac{r}{2}\left(M_{\mathrm{A}}+M_{\mathrm{Hsym}}+3\right)\right] \chi=\frac{1}{2}\left[S_{\mathrm{A}}+S_{\mathrm{Hsym}}\right]
$$

which lifts all three non-continuum species to the same doubler point $2 r / a$.

Evidently, the attractive feature of these operators is that the matrices are just of size $N_{c} N \times N_{c} N$, and still only 1 or 2 species survive in the continuum. The question has been raised whether the remaining symmetries of (1) or (2, 3, 4) are sufficient for taking the continuum limit (at a fixed pion mass) by tuning only the standard (relevant) mass term or whether other (relevant, marginal or irrelevant) operators need to be tuned simultaneously [11 14]. In this paper we investigate the eigenvalue spectra of these operators on interacting backgrounds and the chiralities (with respect to $\gamma_{5} \otimes 1,1 \otimes \xi_{5}$ and $\gamma_{5} \otimes \xi_{5}$ ) of their eigenmodes. The idea behind is that the willingness or reluctance of the "bellies" to open up (and hence separate the branches) is indicative of how severe the fermionic operator mixing is - in the Wilson case both 
link smearing and including the clover term tend to clear the first eigenvalue belly, since they suppress mixing between the dimension 5 Laplacian and the dimension 3 mass operator.

In the proposals [9, 10] (and in [12,17]) a notation is used which obscures the link to the original work [8]. This is why Sec.2 contains a review of taste non-singlet mass terms using standard staggered notation. The core of the investigation, a look at the staggered eigenvalues and chiralities, is presented in Sec.3. A brief discussion of some of the peculiarities of the Symanzik improvement program, when applied to these partly or fully undoubled staggered actions, is given in Sec.4. Results where these taste-split staggered actions serve as kernel to the overlap procedure are shown in Sec.5. A comparison to the eigenvalue spectra of Wilsontype actions is found in Sec.6. Comments on the breaking of rotational symmetry by the Hoelbling operators (2, 3, 4) are arranged in Sec.7, Finally, Sec.8 contains a summary.

\section{Review of staggered mass terms}

Define the Golterman-Smit staggered phase factors [8]

$$
\eta_{\mu}(x)=(-1)^{\sum_{\nu<\mu} x_{\nu}}, \quad \zeta_{\mu}(x)=(-1)^{\sum_{\mu<\nu} x_{\nu}}
$$

which multiply to give

$$
\eta_{5}(x)=\eta_{1} \eta_{2} \eta_{3} \eta_{4}=(-1)^{x_{1}+x_{3}}, \quad \zeta_{5}(x)=\zeta_{1} \zeta_{2} \zeta_{3} \zeta_{4}=(-1)^{x_{2}+x_{4}}
$$

respectively. The product of the latter two phase factors reads (throughout the spinor $\otimes$ taste identification holds up to $O(a)$ corrections [6 8])

$$
\epsilon(x)=\eta_{5} \zeta_{5}=(-1)^{x_{1}+x_{2}+x_{3}+x_{4}} \longleftrightarrow \gamma_{5} \otimes \xi_{5}
$$

and induces the $U(1)_{\epsilon}$-symmetry and $\epsilon$-hermiticity $\epsilon(x) D_{\mathrm{S}}(x, y) \epsilon(y)=D_{\mathrm{S}}^{\dagger}(x, y)=\left[D_{\mathrm{S}}(y, x)\right]^{\dagger}$ of the massless staggered action. After being lifted to a site-diagonal operator

$$
\epsilon(x, y)=\Gamma_{55}(x, y)=(-1)^{x_{1}+x_{2}+x_{3}+x_{4}} \delta_{x, y}
$$

it couples to the $\gamma_{5} \otimes \xi_{5}$ "Goldstone" state when used as an interpolating field [8].

The $\left(\gamma_{\mu} \otimes 1\right)$ and $\left(\gamma_{5} \otimes 1\right)$ "taste singlet" operators are defined by

$$
\begin{aligned}
\Gamma_{\mu 0}(x, y) & \equiv \Gamma_{\mu}(x, y)=\frac{1}{2} \eta_{\mu}(x)\left[U_{\mu}(x) \delta_{x+\hat{\mu}, y}+U_{\mu}^{\dagger}(x-\hat{\mu}) \delta_{x-\hat{\mu}, y}\right] \\
\Gamma_{50}(x, y) & \equiv \Gamma_{5}(x, y)=\frac{1}{4 !} \sum_{\text {perm }} \epsilon_{\mathrm{perm}} \Gamma_{1} \Gamma_{2} \Gamma_{3} \Gamma_{4}
\end{aligned}
$$

and the $\left(1 \otimes \xi_{\mu}\right)$ and $\left(1 \otimes \xi_{5}\right)$ "spinor singlet" operators are defined by

$$
\begin{aligned}
\Gamma_{0 \mu}(x, y) & \equiv \Xi_{\mu}(x, y)=\frac{1}{2} \zeta_{\mu}(x)\left[U_{\mu}(x) \delta_{x+\hat{\mu}, y}+U_{\mu}^{\dagger}(x-\hat{\mu}) \delta_{x-\hat{\mu}, y}\right] \\
\Gamma_{05}(x, y) & \equiv \Xi_{5}(x, y)=\frac{1}{4 !} \sum_{\text {perm }} \epsilon_{\text {perm }} \Xi_{1} \Xi_{2} \Xi_{3} \Xi_{4}
\end{aligned}
$$

with the consequence that both $\Gamma_{50}$ and $\Gamma_{05}$ are 4-hop operators. Furthermore, the latter two operators relate to each other by a simple $\Gamma_{55}$ operation (either from the left or from the right). 
In practice it can be advantageous to introduce the commutators in spinor and taste space

$$
\begin{aligned}
& \Gamma_{\mu \nu}(x, y) \equiv \frac{\mathrm{i}}{2}\left(\Gamma_{\mu} \Gamma_{\nu}-\Gamma_{\nu} \Gamma_{\mu}\right) \quad \longleftrightarrow \quad \gamma_{\mu \nu} \otimes 1 \\
& \Xi_{\mu \nu}(x, y) \equiv \frac{\mathrm{i}}{2}\left(\Xi_{\mu} \Xi_{\nu}-\Xi_{\nu} \Xi_{\mu}\right) \quad \longleftrightarrow \quad 1 \otimes \xi_{\mu \nu}
\end{aligned}
$$

respectively, with $\gamma_{\mu \nu} \equiv \frac{\mathrm{i}}{2}\left[\gamma_{\mu}, \gamma_{\nu}\right]$ known as $\sigma_{\mu \nu}$ and $\xi_{\mu \nu} \equiv \frac{\mathrm{i}}{2}\left[\xi_{\mu}, \xi_{\nu}\right]$. Based on these one finds

$$
\begin{aligned}
& \Gamma_{50}(x, y) \simeq-\frac{1}{6}\left(\Gamma_{12} \Gamma_{34}-\Gamma_{13} \Gamma_{24}+\Gamma_{14} \Gamma_{23}+\Gamma_{23} \Gamma_{14}-\Gamma_{24} \Gamma_{13}+\Gamma_{34} \Gamma_{12}\right) \\
& \Gamma_{05}(x, y) \simeq-\frac{1}{6}\left(\Xi_{12} \Xi_{34}-\Xi_{13} \Xi_{24}+\Xi_{14} \Xi_{23}+\Xi_{23} \Xi_{14}-\Xi_{24} \Xi_{13}+\Xi_{34} \Xi_{12}\right)
\end{aligned}
$$

where the near-equality is exact if no $\mathrm{SU}(3)$ backprojection in intermediate steps is applied. The main advantage of the form (15 16) is that one can apply $\mathrm{SU}(3)$ backprojection twice, in each case the argument being a sum over products of just two color matrices.

Following Golterman and Smit [8], everything which is a singlet in spinor space is called a "mass term". This leads to the categorization of potential mass terms as

$$
\begin{array}{rlll}
1 \otimes 1 & \longleftrightarrow & 1 & \text { (0-hop, taste-scalar) } \\
1 \otimes \xi_{\mu} & \longleftrightarrow \Xi_{\mu} & \text { (1-hop, taste-vector) } \\
1 \otimes \xi_{\mu \nu} & \longleftrightarrow \Xi_{\mu \nu} & \text { (2-hop, taste-tensor) } \\
1 \otimes \mathrm{i} \xi_{\mu} \xi_{5} & \longleftrightarrow \mathrm{i} \Xi_{\mu} \Xi_{5} & \text { (3-hop, taste-pseudovector) } \\
1 \otimes \xi_{5} & \longleftrightarrow & \Xi_{5} & \text { (4-hop, taste-pseudoscalar) }
\end{array}
$$

in terms of hermitian operators. As was pointed out in [12], an acceptable mass term is $1 \otimes 1$, $1 \otimes \xi_{\mu \nu}, 1 \otimes \xi_{5}$ or a combination thereof. In this terminology the Adams term in (1) is the taste-pseudoscalar mass $\Gamma_{05}=\Xi_{5}$. And the Hoelbling terms in (2,3) represent unsymmetrized and symmetrized versions of the taste-tensor mass $\Xi_{\mu \nu}$, respectively, i.e. $M_{\text {Hori }}=\Xi_{12}$ [to be used together with $M_{\mathrm{A}}$, see [2] ] and $M_{\mathrm{Hsym}}=\left(\Xi_{12}+\Xi_{34}+\Xi_{13}-\Xi_{24}+\Xi_{14}+\Xi_{23}\right) / \sqrt{3}$ [to be used alone, see (3)]. The operator (4), finally, involves all three types of valid mass terms.

Note that there is some freedom in the definition of the operators (1, 4) and of the taste non-singlet mass terms. For instance with the Adams operator as defined in (1) and the choice $M_{A}=\gamma_{5} \otimes \xi_{5}$ it follows that the physical branch has $\xi_{5}=-1$. Hence, with this convention the actions of $\gamma_{5} \otimes 1$ and $\gamma_{5} \otimes \xi_{5}$, in the physical branch, on a topological mode will differ in sign.

\section{$3 \quad$ Eigenvalues and chiralities}

The eigenvalue spectrum on a thermalized gauge background is a key to get an impression of how suitable a given fermion discretization is in practical terms. An issue with staggered fermions is their sensitivity to the topological charge of the gauge configuration [18 21].

The $\epsilon$-hermiticity ensures that the eigenvalues of the massless operator occur in pairs $\pm \mathrm{i} \lambda$ on the imaginary axis. There are no exact zero-modes of $D_{\mathrm{S}}$; instead $4|q|$ would-be zero-modes show up. Both their separation from the non-topological modes and their chiralities (see below) depend on how much the staggered action is "improved" through link smearing. 
4D: $N C=3, \beta=5.8, L=6, T=6,|q|=1, C \_S W=0$

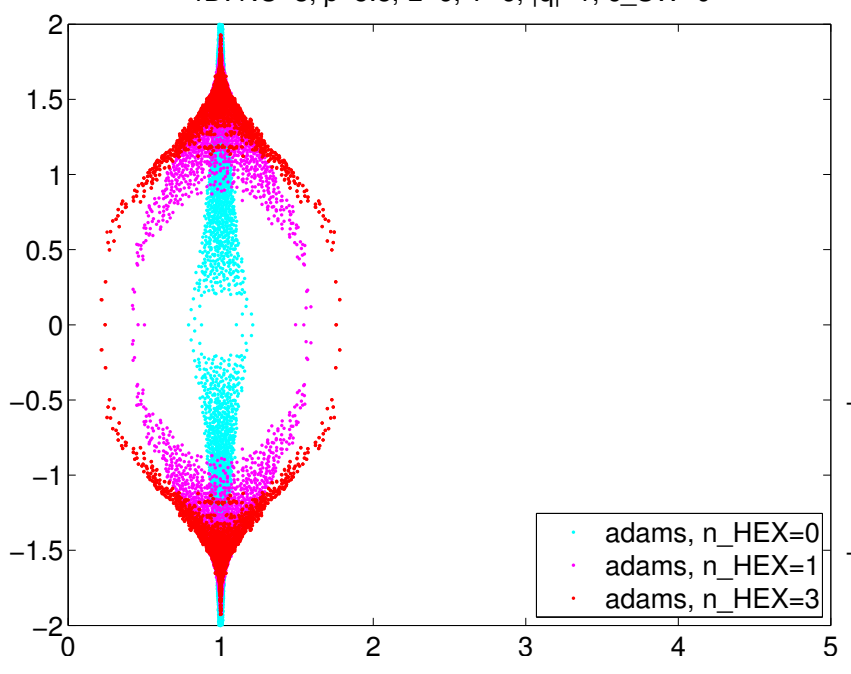

4D: $N C=3, \beta=5.8, L=6, T=6,|q|=1, C \_S W=0$

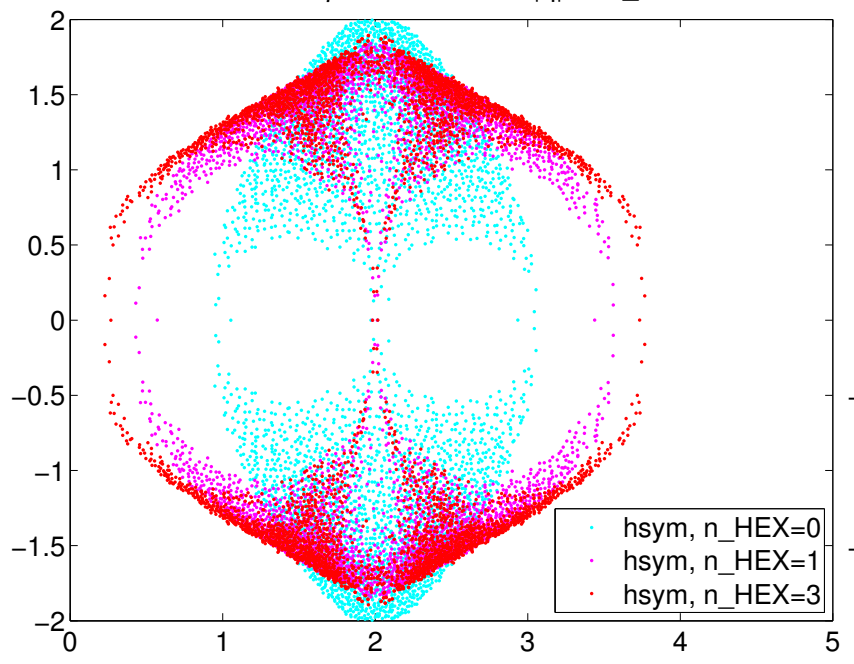

4D: $N C=3, \beta=5.8, L=6, T=6,|q|=1, c \_S W=0$

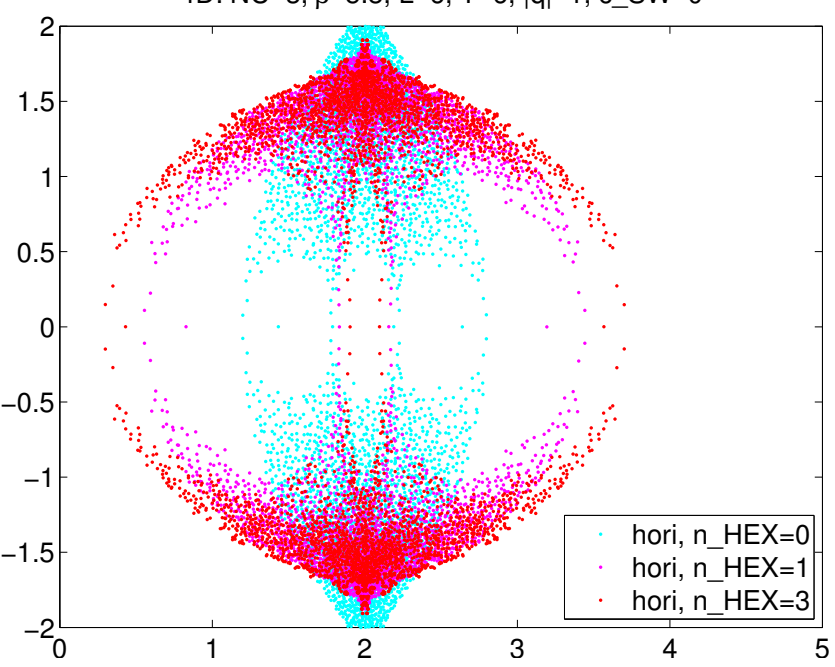

4D: $N C=3, \beta=5.8, L=6, T=6,|q|=1, C \_S W=0$

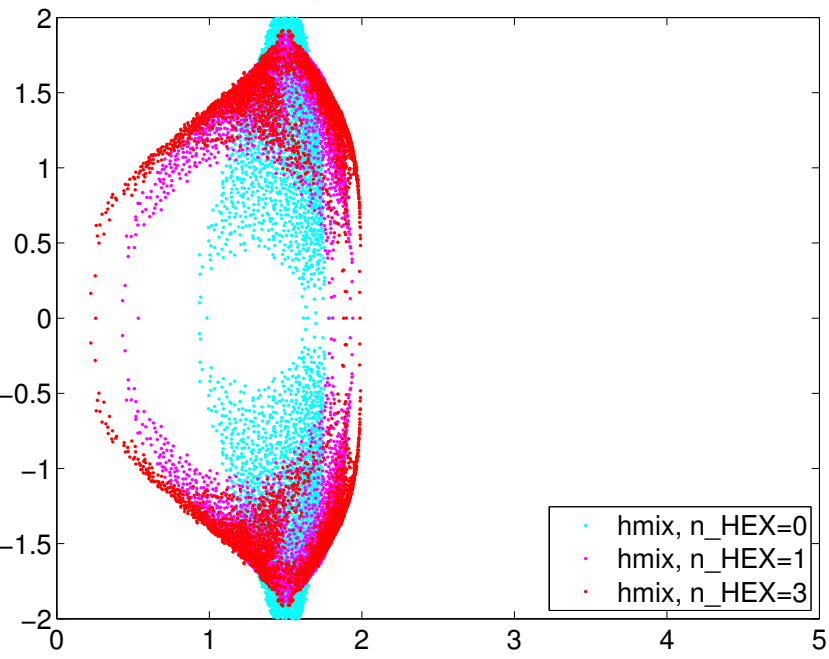

Figure 1: Eigenvalue spectra of the four unimproved operators $\left(c_{\mathrm{SW}}=0\right)$ with $r=1$ at $m=0$ for up to three levels of HEX smearing - the smearing seems essential for the bellies to form.

Fig. 1 displays the eigenvalue spectra of the operators $(1-4)$ on a $6^{4}$ lattice (generated with $\beta=5.8$ Wilson glue) of unit topological charge, $|q|=1$. The fermion boundary conditions are periodic in all directions; with antiperiodic boundary conditions in the 4-direction mild changes would occur in regions of large (positive or negative) imaginary part. Throughout, we use gauge links $V_{\mu}(x)$ which have undergone 0,1 , or 3 levels of HEX smearing [22]. Backprojection of the mean of the $n$-hop paths to $\mathrm{SU}(3)$ could be applied, but in the present work this is not done.

Our first observation in these plots is that without link-smearing the "bellies" have a hard time opening up (as was already observed in [12]), but the situation improves considerably upon applying 1 or 3 steps of smearing. All operators have a bare (taste-scalar) mass $m=0$, but their physical (left-most) branches cross the real axis at distinctively non-zero values. This is a sign of additive mass renormalization, and the plots show that the smearing reduces this effect significantly. Last but not least the physical branches have one [or two for (1), since this operator is doubled] exactly real modes which tend to get "soaked into the belly" even for 
4D: $N C=3, \beta=5.8, L=6, T=6,|q|=1$, c_SW=0

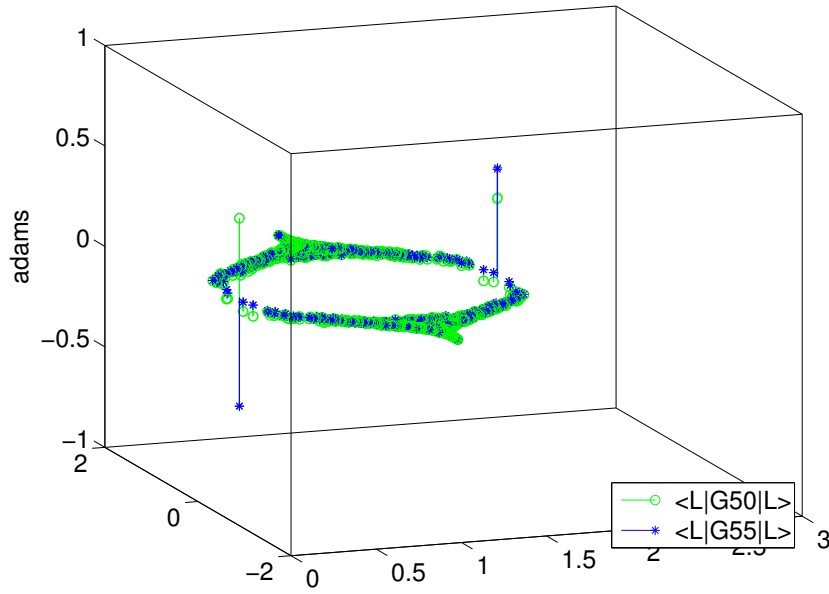

4D: $N C=3, \beta=5.8, L=6, T=6,|q|=1, c \_S W=0$

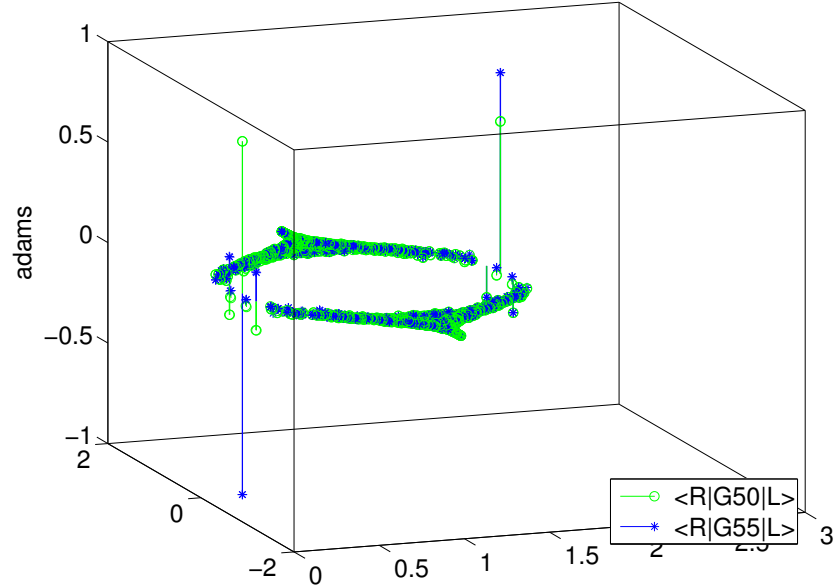

4D: $N C=3, \beta=5.8, L=6, T=6,|q|=1$, c_SW=0

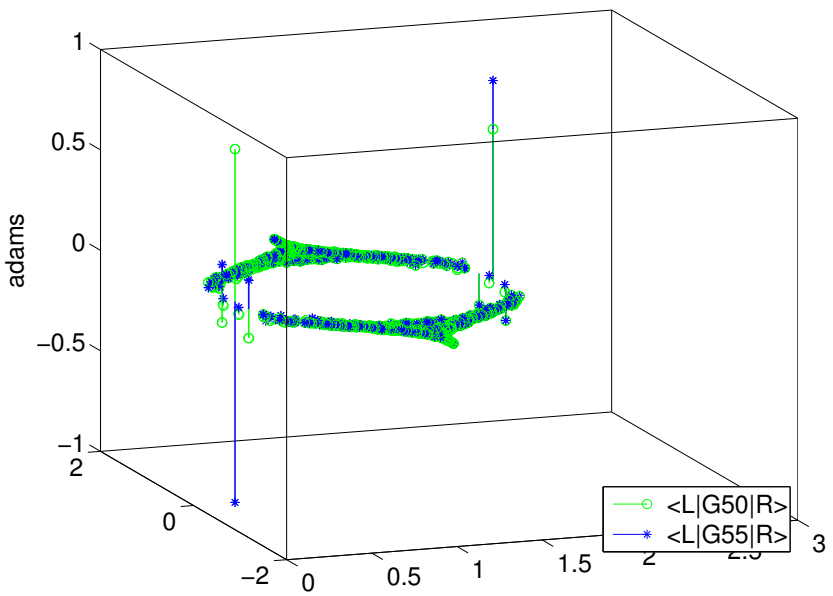

4D: $N C=3, \beta=5.8, L=6, T=6,|q|=1, c \_S W=0$

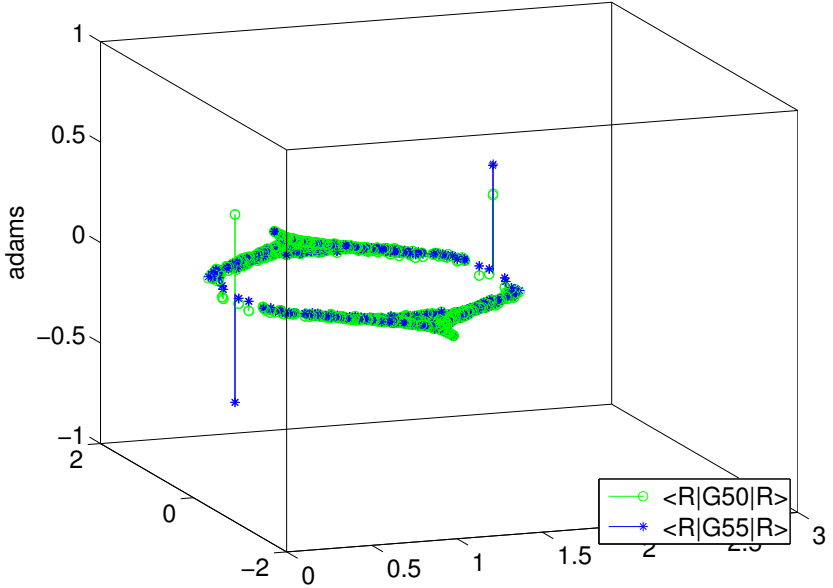

Figure 2: Needle plots of the four $L / R$-chiralities of the unimproved Adams operator $\left(c_{\mathrm{SW}}=0\right)$ with respect to $\Gamma_{50}$ (green circles) or $\Gamma_{55}=\epsilon$ (blue stars), with three HEX smearings.

the higher smearing levels. And of course all spectra are symmetric with respect to the real axis, which implies that the determinant is real (this is a consequence of the requirement of $\epsilon$-hermiticity which ruled out two possible mass terms, cf. Sec.21).

The next step is to look at the chiralities of the pertinent eigenmodes, i.e. the values of the operators $\Gamma_{50}, \Gamma_{05}$ and $\Gamma_{55}$ when sandwiched between the staggered eigenmodes. The standard operator $D_{\mathrm{S}}$ or $D_{\mathrm{S}}+m$ is a normal operator, i.e. $\left[D_{\mathrm{S}}, D_{\mathrm{S}}^{\dagger}\right]=0$, and only $\Gamma_{50}$ is sensitive to the topological charge of the background (see below). The taste-split staggered operators (14 4 are non-normal operators, i.e. $\left[D, D^{\dagger}\right] \neq 0$. This implies that the left-eigenvectors, which satisfy $\left\langle L_{i}\right| D=\left\langle L_{i}\right| \lambda_{i}$, are not just the hermitian conjugates of the right-eigenvectors, which satisfy $D\left|R_{i}\right\rangle=\lambda_{i}\left|R_{i}\right\rangle$, though they share the eigenvalue $\lambda_{i}$. The situation is now analogous to that of the Wilson operator which is also non-normal [23]. This implies that for a given sandwich operator $\Gamma$ there are four chiralities, $\left\langle L_{i}|\Gamma| L_{i}\right\rangle,\left\langle L_{i}|\Gamma| R_{i}\right\rangle,\left\langle R_{i}|\Gamma| L_{i}\right\rangle,\left\langle R_{i}|\Gamma| R_{i}\right\rangle$, where $\left|L_{i}\right\rangle$ is the hermitian conjugate of the (normalized) left-eigenvector $\left\langle L_{i}\right|$, and $\left\langle R_{i}\right|$ is the hermitian conjugate of the (normalized) right-eigenvector $\left|R_{i}\right\rangle$, with $i$ running over all modes.

Fig.2 displays these four options with the sandwich operators $\Gamma_{50}$ and $\Gamma_{55}$ for the eigenmodes 
4D: $N C=3, \beta=5.8, L=6, T=6,|q|=1$

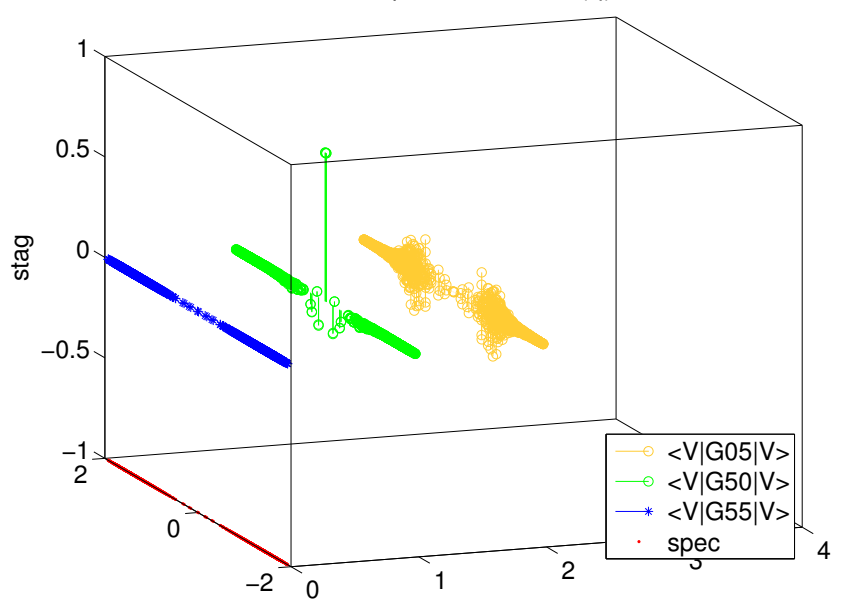

4D: $N C=3, \beta=5.8, L=6, T=6,|q|=1$, c_SW=0

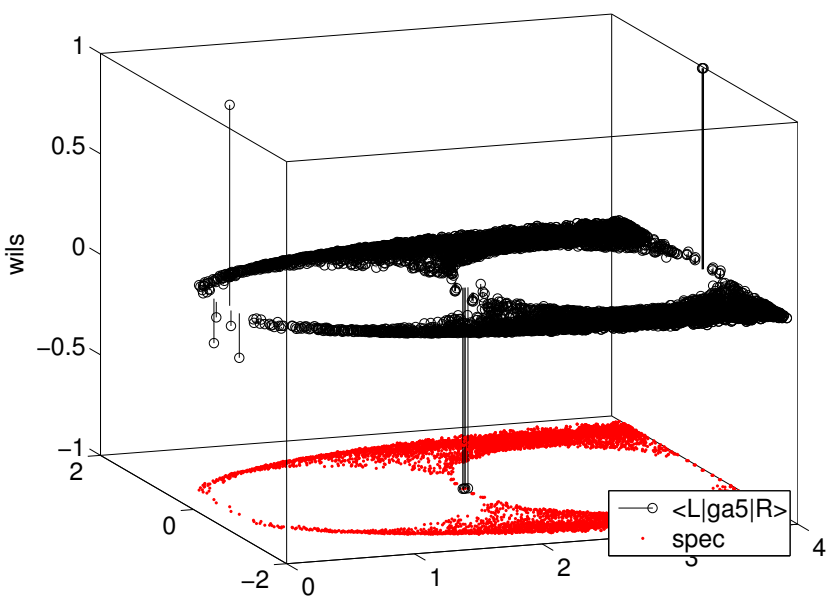

Figure 3: Chiralities of the standard staggered action and of the unimproved Wilson operator. For better visibility the results for $\left\langle.\left|\Gamma_{50}\right|.\right\rangle$ and $\left\langle.\left|\Gamma_{05}\right|.\right\rangle$ are displaced in the real direction.

of the Adams operator (1). The chiralities are plotted in the $z$-direction above the respective eigenvalue $\lambda=x+\mathrm{i} y$ in the complex plane. The four options collapse effectively into two, as it must be, due to the $\epsilon$-hermiticity of the operator. The $\langle L|\Gamma| L\rangle$ or $\langle R|\Gamma| R\rangle$ option shows two distinctively non-zero physical modes (on a configuration with $|q|=1$ ), depicted at the position of the two exactly real eigenvalues, both for $\Gamma=\Gamma_{50}$ and $\Gamma=\Gamma_{55}$ (peeking into the $+z$ and $-z$ directions, respectively). With the $\langle L|\Gamma| R\rangle$ or $\langle R|\Gamma| L\rangle$ option the chiralities tend to be even more pronounced, and in either case it holds that the magnitude of $\left\langle\cdot\left|\Gamma_{55}\right|.\right\rangle$ exceeds the magnitude of $\left\langle.\left|\Gamma_{50}\right|.\right\rangle$. Finally there is an equal number of chiral modes in the unphysical branch (near $x=2$ ) with identical orientation for $\Gamma_{50}$ but opposite orientation for $\Gamma_{55}$ (in accordance with Karsten-Smit [18]). The most surprising lesson is that both $\Gamma_{50}$ and $\Gamma_{55}$ prove sensitive to the topology of the gauge background; this happens in sharp contrast to the standard staggered case (cf. next paragraph). And the recommendation of 23] that for any $\Gamma$ one should focus on the $\langle L|\Gamma| R\rangle$ option continues to be valid.

Fig. 3 displays - for comparison - the chiralities of the standard staggered action and of the Wilson action on the same $|q|=1$ gauge background (only half of the spectrum is shown in the latter case). In the staggered case there is only one type of eigenmode, due to $\left\langle V_{i}|=| V_{i}\right\rangle^{\dagger}$, while in the Wilson case only $\left\langle L_{i}\left|\gamma_{5}\right| R_{i}\right\rangle$ is shown. In the staggered case $\left\langle V_{i}\left|\Gamma_{55}\right| V_{i}\right\rangle$ is absolutely flat (as required by $\left\{\epsilon, D_{\mathrm{S}}\right\}=0$ ), while $\left\langle V_{i}\left|\Gamma_{50}\right| V_{i}\right\rangle$ (plotted at $x=1$ ) has 4 modes which peek upwards, and $\left\langle V_{i}\left|\Gamma_{05}\right| V_{i}\right\rangle$ (plotted at $x=2$ ) is wiggly but not sensitive to topology. The Wilson operator shows the expected behavior - one chiral mode in the physical branch, four oppositely oriented modes near $x=2$, six originally oriented modes near $x=4$, four oppositely oriented modes near $x=6$ (not shown) and one originally oriented mode near $x=8$ (not shown).

Fig. 4 displays the $\left\langle L\left|\Gamma_{55}\right| R\right\rangle$ chiralities for the four operators (1-4) above their eigenvalues. The Adams operator has two chiral modes in the physical branch and two unphysical modes with opposite orientation. The unsymmetrized Hoelbling operator has one chiral mode which is physical, two modes of unequal orientation near $x=2$ and one oppositely oriented mode near $x=4$. The symmetrized version has one chiral mode which is physical, two oppositely oriented modes near $x=2$ and one mode with original orientation near $x=4$. The mixed operator (4), finally, has one chiral mode which is physical and three unphysical modes near $x=2$ (two of 
4D: $N C=3, \beta=5.8, L=6, T=6,|q|=1$, c_SW=0

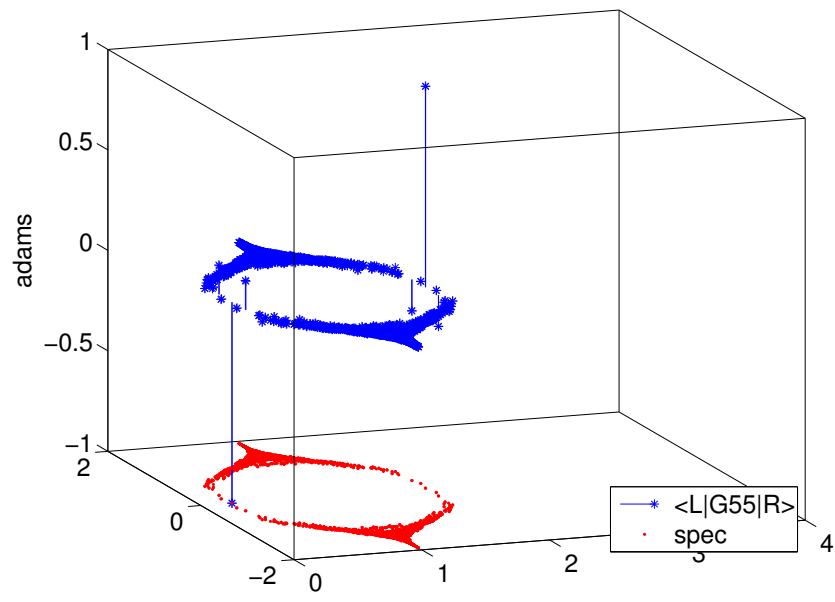

4D: $N C=3, \beta=5.8, L=6, T=6,|q|=1, c \_S W=0$

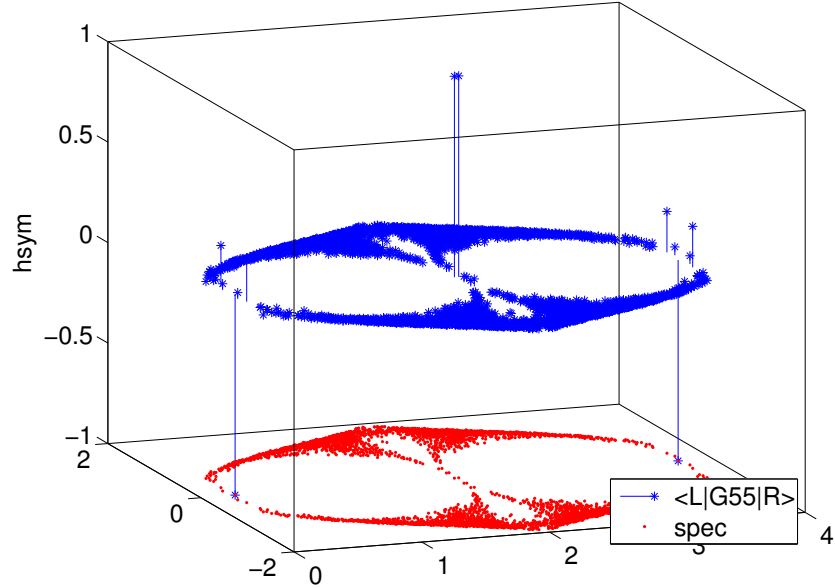

4D: $N C=3, \beta=5.8, L=6, T=6,|q|=1$, c_SW=0

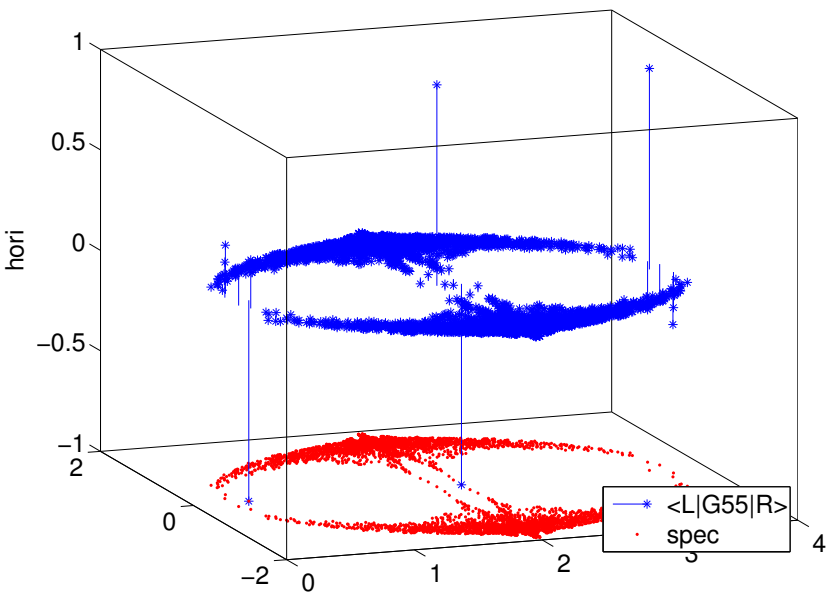

4D: $N C=3, \beta=5.8, L=6, T=6,|q|=1, c \_S W=0$

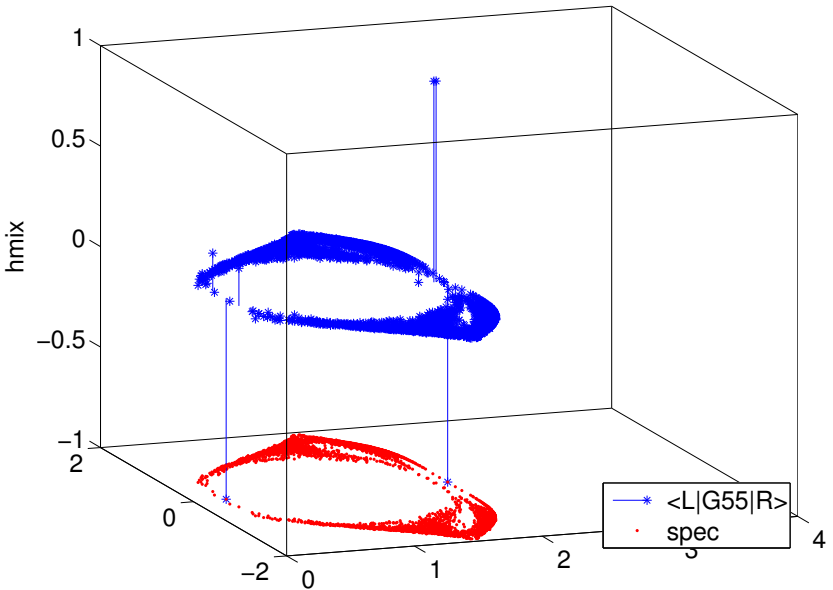

Figure 4: Needle plots of the chiralities with respect to $\Gamma_{55}$ of the unimproved operators $\left(c_{\mathrm{SW}}=\right.$ $0)$ above their eigenvalues. The LR-definition of $\left\langle.\left|\Gamma_{55}\right|.\right\rangle$ and three HEX smearings are used.

which are antiparallel, one of which is parallel to the chirality of the physical mode). In each case it was checked that the choice $\Gamma_{50}$ (instead of $\Gamma_{55}$ ) or the naive option $\langle R|| R$.$\rangle (instead of$ $\langle L|| R\rangle$.$) bring exactly the kind of change that one would anticipate from Fig.2.$

\section{Results with Symanzik improvement}

So far the investigation of the eigenvalues and eigenmode chiralities of the taste-split staggered actions (14 4) leaves us with the impression that they are technically rather close to the usual Wilson action - with chiral symmetry breaking and all the consequences of non-normality, e.g. $\langle L|$ and $|R\rangle$ eigenmodes. This raises the question whether some of the remedies which have proven useful in taming these effects with the Wilson action might be taken over and/or adapted to these taste-split staggered actions. The most successful remedies were link smearing, clover improvement and the overlap procedure - they all mitigate (in the last case eliminate) the effects of chiral symmetry breaking, and they can be used in various combinations.

That link smearing proves useful for staggered actions with taste non-singlet mass terms 
4D: $N C=3, \beta=5.8, L=6, T=6,|q|=1, C \_S W=1$

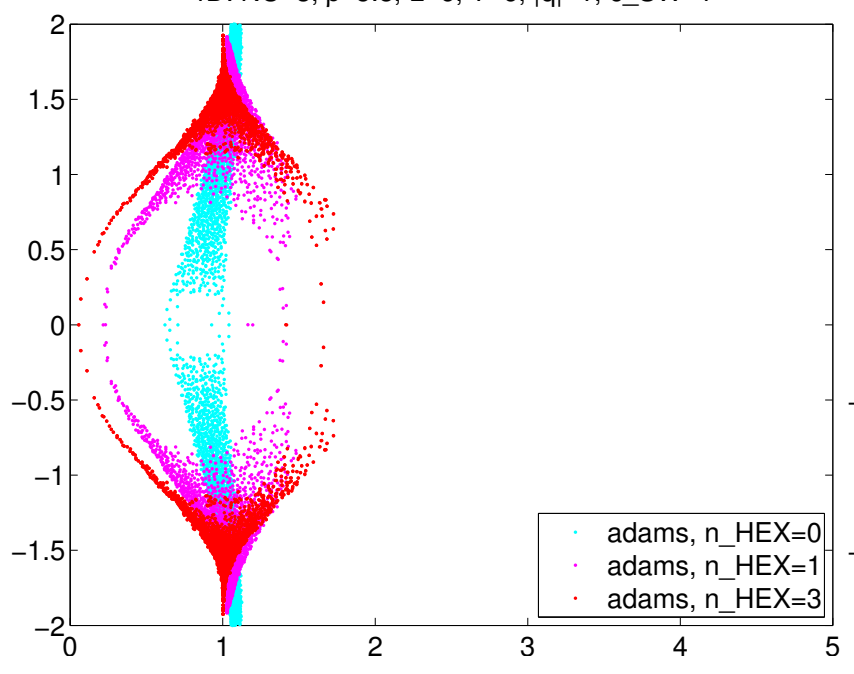

4D: $N C=3, \beta=5.8, L=6, T=6,|q|=1, C \_S W=1$

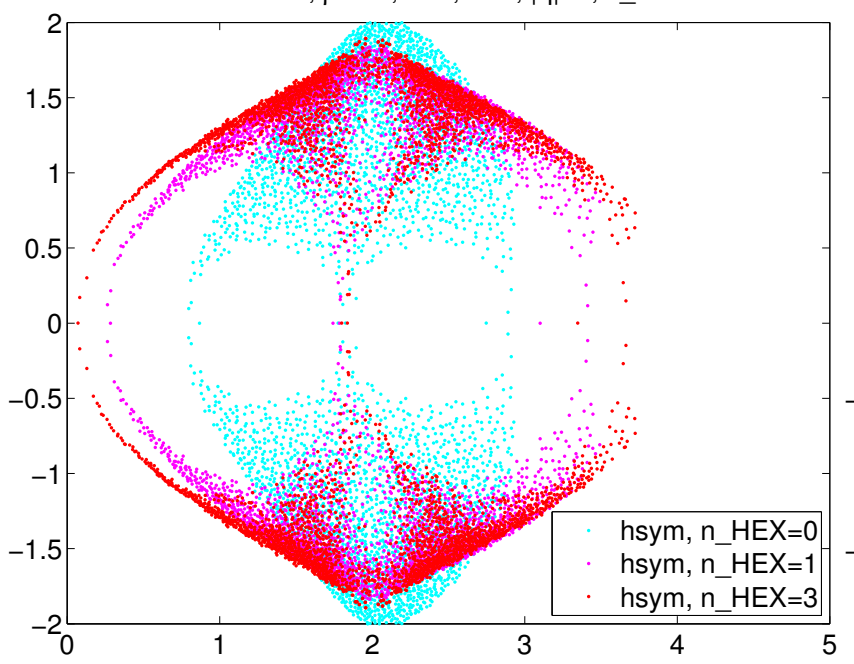

4D: $N C=3, \beta=5.8, L=6, T=6,|q|=1$, c_SW=1

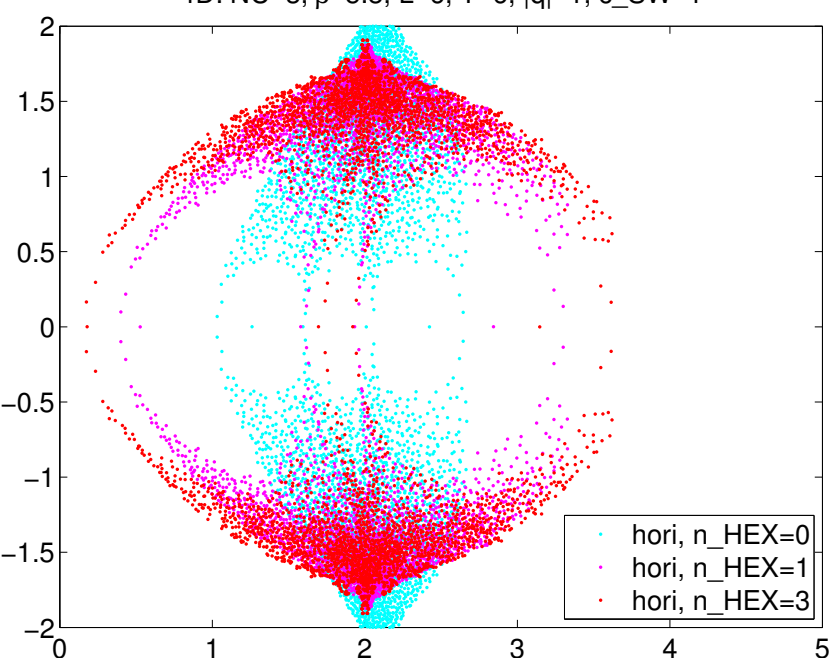

4D: $N C=3, \beta=5.8, L=6, T=6,|q|=1, C \_S W=1$

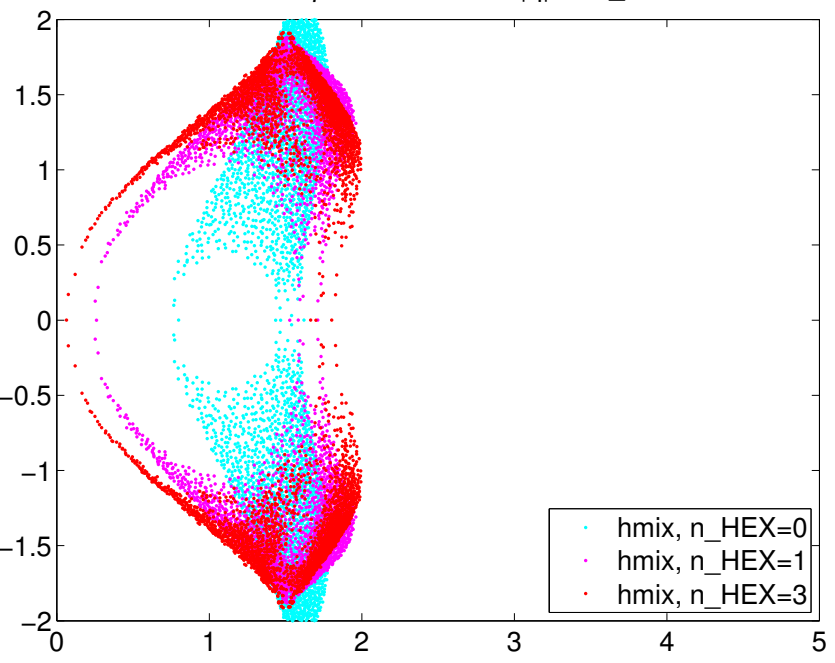

Figure 5: Eigenvalue spectra of the four improved operators $\left(c_{\mathrm{SW}}=1\right)$ at $m=0$ for up to three levels of HEX smearing - the smearing seems essential for the bellies to form.

has already been shown in the previous section - in Fig.1 a clear improvement of the overall properties of the eigenvalue spectra with an increased number of smearing steps is evident (and consequently in Figs. 2, 3, 4 only the results for three smearing steps were displayed).

The next step is Symanzik improvement [24]. In principle this is a program to be carried out in strict analogy to the Wilson case - one writes down a complete list of operators, ordered by their mass dimensions, and eliminates those which are forbidden by symmetries or redundant by the equation of motion. In the Wilson case one finds that only the $d=5$ term $\bar{\psi} \gamma_{\mu \nu} F_{\mu \nu} \psi$ needs to be added to the action [25], with a tuned coefficient $c_{\mathrm{SW}} / 2$ in front to cancel all $O(a)$ cut-off effects (thus eliminating all signs of chiral symmetry breaking to this order) [26, 27].

With staggered fermions the situation is a bit more delicate, due to the "staggered" representation of the spinor degrees of freedom. The point is that the operators $\Gamma_{\mu} \equiv \eta_{\mu} C_{\mu}$ and $\Xi_{\mu} \equiv \zeta_{\mu} C_{\mu}$ formally leave the mass dimension $d$ of a fermion bilinear invariant, but they do involve a fermion hop [made gauge invariant through a link $U_{\mu}(x)$ or $V_{\mu}(x)$ ]. In other words, the 
4D: $N C=3, \beta=5.8, L=6, T=6,|q|=1, c \_S W=1$

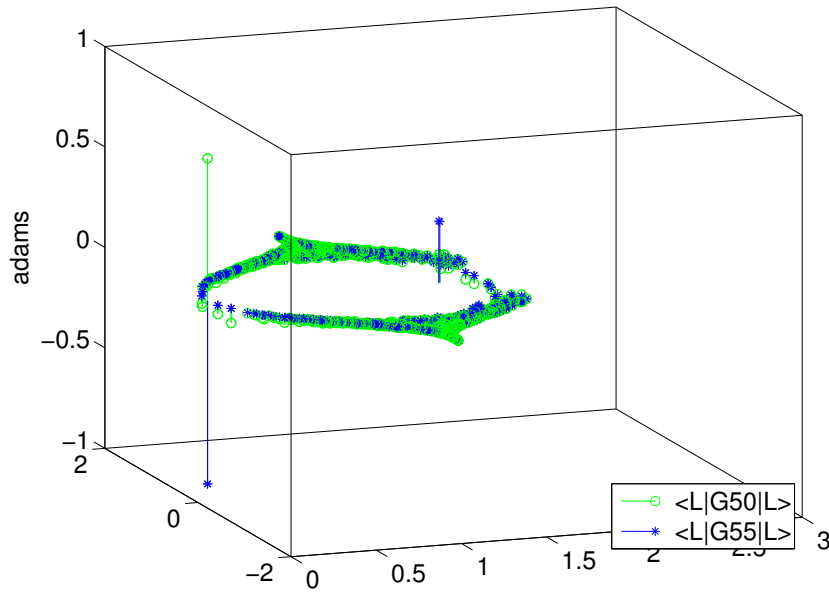

4D: $N C=3, \beta=5.8, L=6, T=6,|q|=1, c \_S W=1$

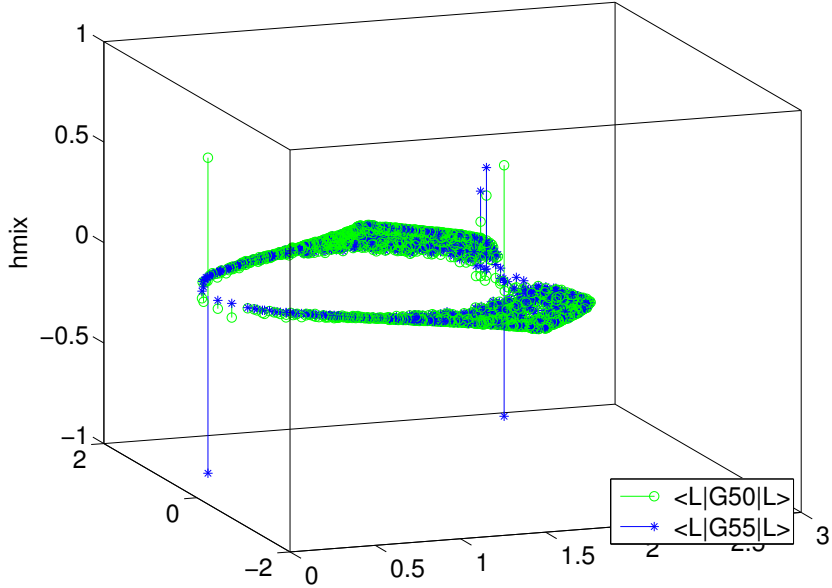

4D: $N C=3, \beta=5.8, L=6, T=6,|q|=1$, c_SW=1

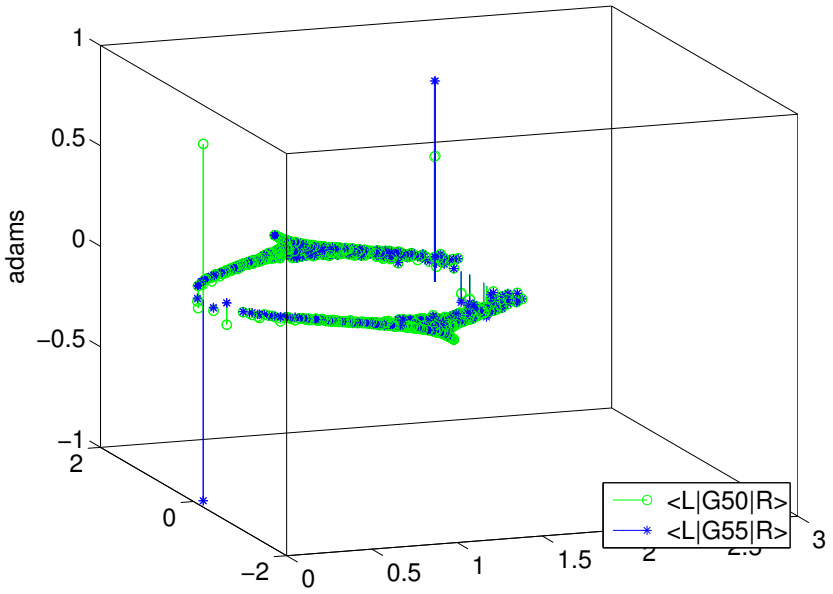

4D: $N C=3, \beta=5.8, L=6, T=6,|q|=1, c \_S W=1$

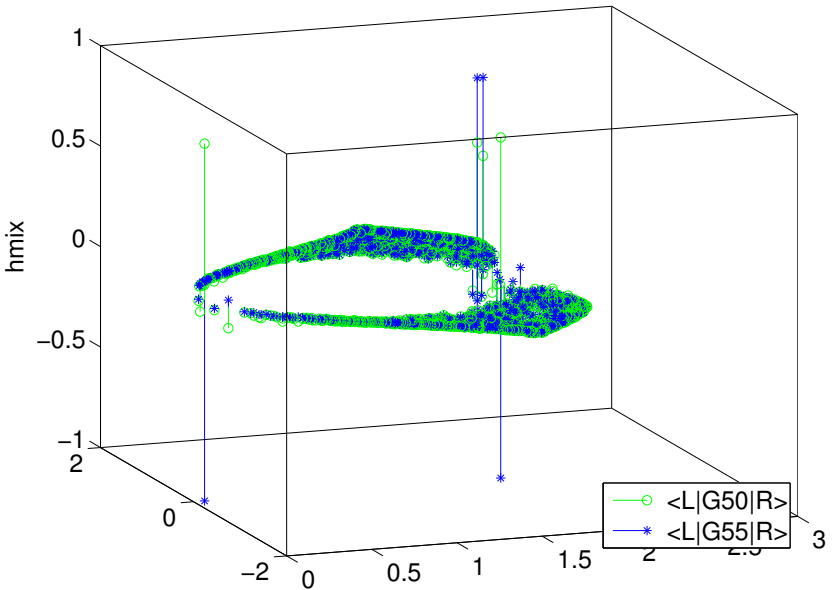

Figure 6: Needle plots of the LL (left) and LR (right) chiralities of the Adams operator (1) (top) and of the mixed operator (4) (bottom), with respect to $\Gamma_{50}$ (green circles) or $\Gamma_{55}=\epsilon$ (blue stars), with Symanzik improvement $\left(c_{\mathrm{SW}}=1\right)$ and three HEX smearings.

strict coincidence of the mass dimension of the operator (as is relevant for the Symanzik analysis) and the maximum number of hops (that affects the strength of the mixing or the "noise" of the operator) is now broken. It seems that this requires a more in-depth analysis [11]. Here we give an incomplete and redundant list of staggered bilinears (with "+h.c." implicit)

$$
\begin{array}{ll}
d=3: & \bar{\psi}\left\{1, \Gamma_{\mu}, \Xi_{\mu}, \Gamma_{\mu}^{2}, \Gamma_{\mu} \Xi_{\mu}, \Xi_{\mu}^{2}, \ldots\right\} \psi \\
d=4: & \bar{\psi}\left\{\eta_{\mu} D_{\mu}, \zeta_{\mu} D_{\mu}, \Gamma_{\mu} D_{\mu}, \Xi_{\mu} D_{\mu}, \ldots\right\} \psi \\
d=5: & \bar{\psi}\left\{D_{\mu}^{2}, \eta_{\mu} D_{\mu}^{2}, \zeta_{\mu} D_{\mu}^{2}, \Gamma_{\mu} D_{\mu}^{2}, \Xi_{\mu} D_{\mu}^{2}, \Gamma_{\mu \nu} F_{\mu \nu}, \Xi_{\mu \nu} F_{\mu \nu}, \ldots\right\} \psi
\end{array}
$$

along with a statement that most of them can be eliminated by means of the arguments mentioned above and the identification of operators which differ only by a factor $\left(1 \otimes \xi_{5}\right)$. The reasoning sketched in App.A suggests that only a single operator needs to be included

$$
-\frac{c_{\mathrm{SW}}}{4}\left\{\Gamma_{12} F_{12}+\ldots+\Gamma_{34} F_{34}+F_{12} \Gamma_{12}+\ldots+F_{34} \Gamma_{34}\right\}=-\frac{c_{\mathrm{SW}}}{4}\left\{\sum_{\mu<\nu} \Gamma_{\mu \nu} F_{\mu \nu}+\text { h.c. }\right\}
$$


4D: $N C=3, \beta=5.8, L=6, T=6,|q|=1, c \_S W=1$

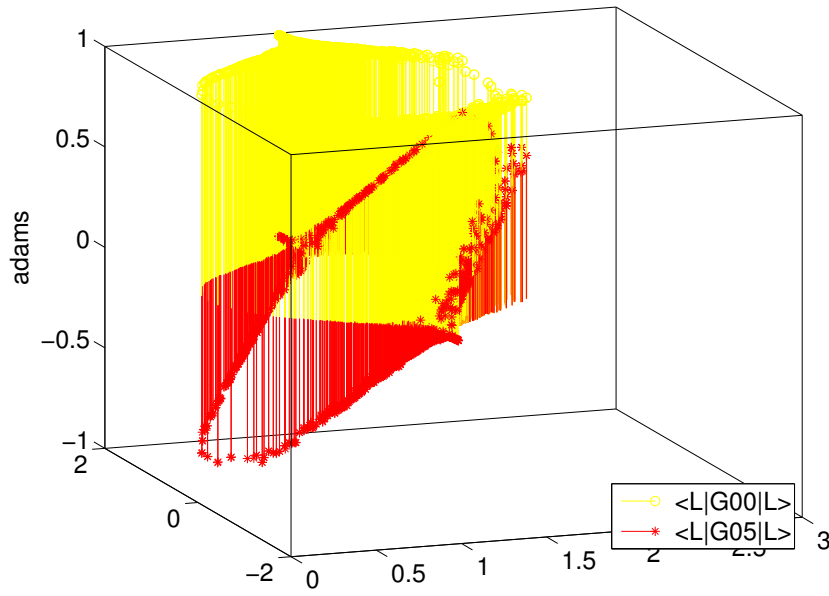

4D: $N C=3, \beta=5.8, L=6, T=6,|q|=1, c \_S W=1$

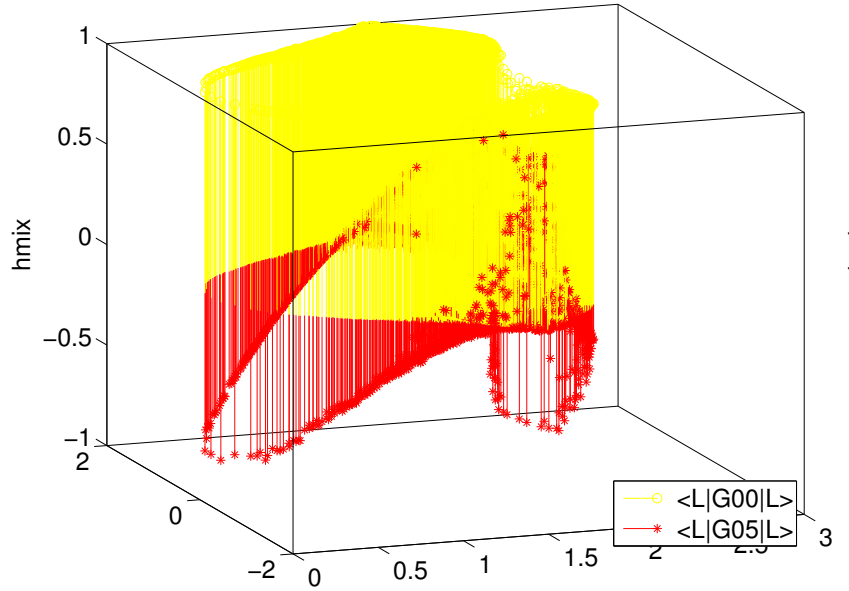

4D: $N C=3, \beta=5.8, L=6, T=6,|q|=1$, c_SW=1

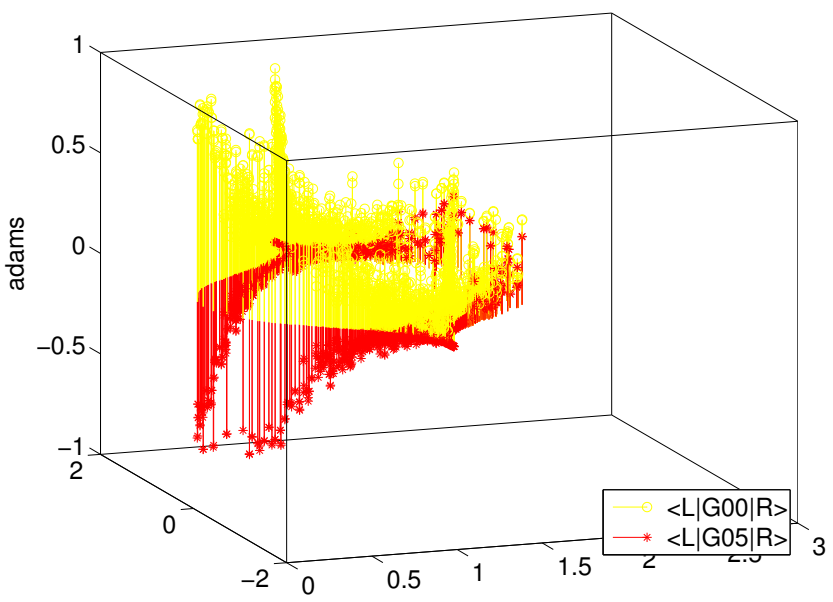

4D: $N C=3, \beta=5.8, L=6, T=6,|q|=1, c \_S W=1$

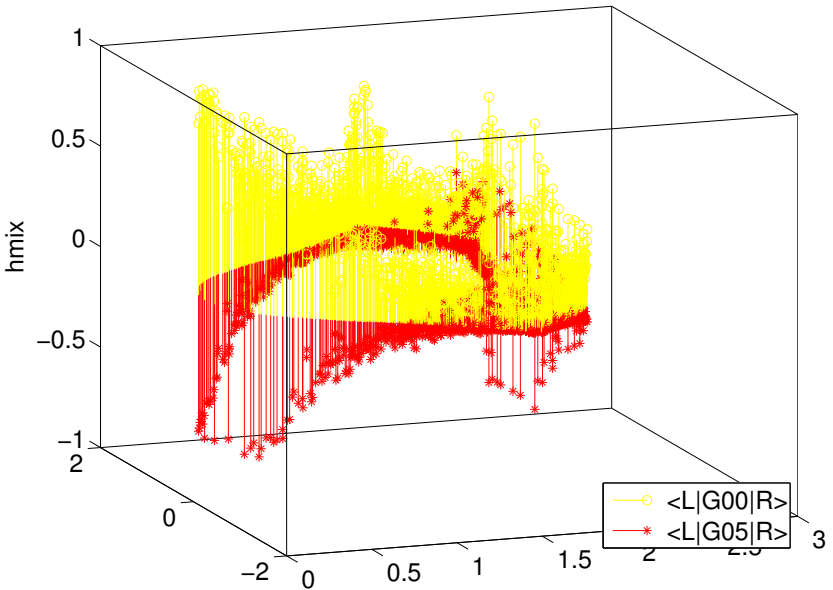

Figure 7: Needle plots of the LL (left) and LR (right) overlaps $\left\langle.\left|\Gamma_{00}\right|.\right\rangle$ (yellow circles) and taste chiralities $\left\langle.\left|\Gamma_{05}\right|.\right\rangle$ (red stars) for the Adams operator (1) (top) and the mixed operator (4) (bottom), with Symanzik improvement $\left(c_{\mathrm{SW}}=1\right)$ and three HEX smearings.

to be compared to $-\frac{c_{\mathrm{SW}}}{2} \sum_{\mu<\nu} \gamma_{\mu \nu} F_{\mu \nu} \delta_{x, y}$ with Wilson fermions (in either case factors are chosen such that $c_{\mathrm{SW}}=1$ at tree level). Note that $F_{\mu \nu}$ is site-diagonal, but not color-diagonal, whereas $\Gamma_{\mu \nu}$ is neither site- nor color-diagonal. The symmetrization in (25) ensures gauge-covariance (in contradistinction to gauge-invariance in the Wilson case) and hermiticity.

Fig. 5 displays the eigenvalues of the four operators (1.40) with the improvement term (25) on the same gauge configuration (with $|q|=1$ ) as in Sec.3. Relative to Fig.11 one finds a clear amelioration of the behavior of the physical (leftmost) branch of eigenvalues; it is now much thinner and the section close to the origin is much more akin to a shifted Ginsparg-Wilson circle. In particular the tendency of the exactly real modes to get "soaked into" the belly is almost removed, except for the unsymmetrized operator (2). Perhaps the most remarkable feature is that for the unsmeared operators the improvement is hardly useful. It takes the combination of smearing and improvement to get a profound effect - in strict analogy to what is observed for the Wilson case [22]. For clarity we add that the links in the operator, in $\Gamma_{\mu \nu}$ and in $F_{\mu \nu}$ have all undergone the same kind of smearing. Finally, let us add that it was explicitly checked 
4D: $N C=3, \beta=5.8, L=6, T=6,|q|=1, c \_S W=1$

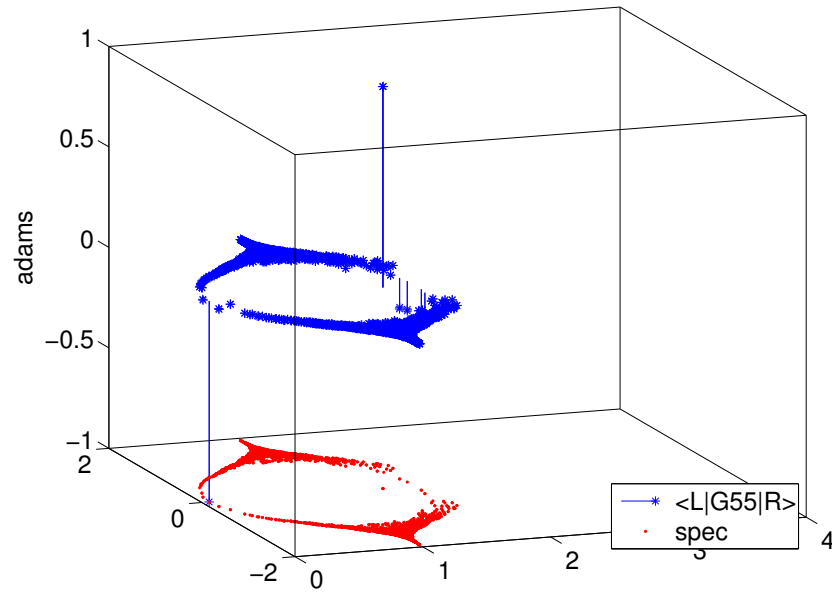

4D: $N C=3, \beta=5.8, L=6, T=6,|q|=1, c \_S W=1$

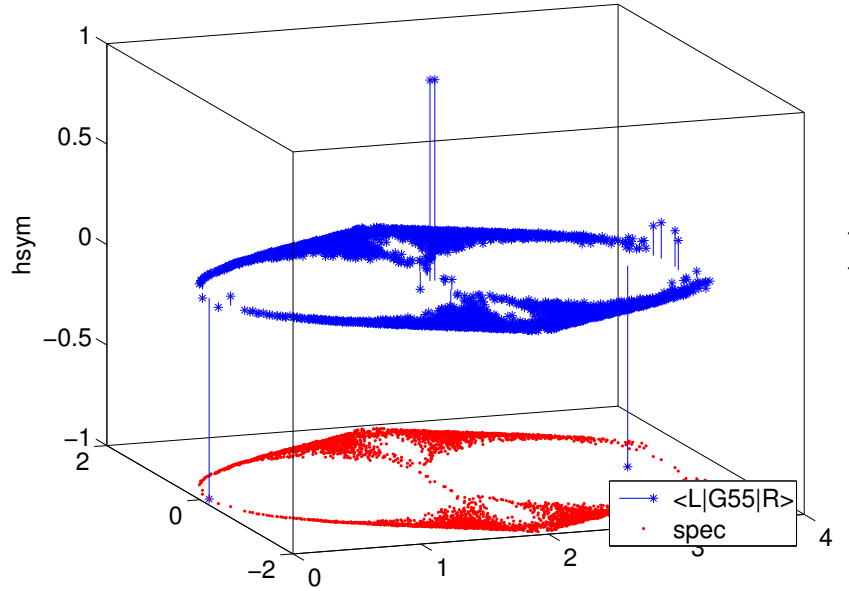

4D: $N C=3, \beta=5.8, L=6, T=6,|q|=1$, c_SW=1

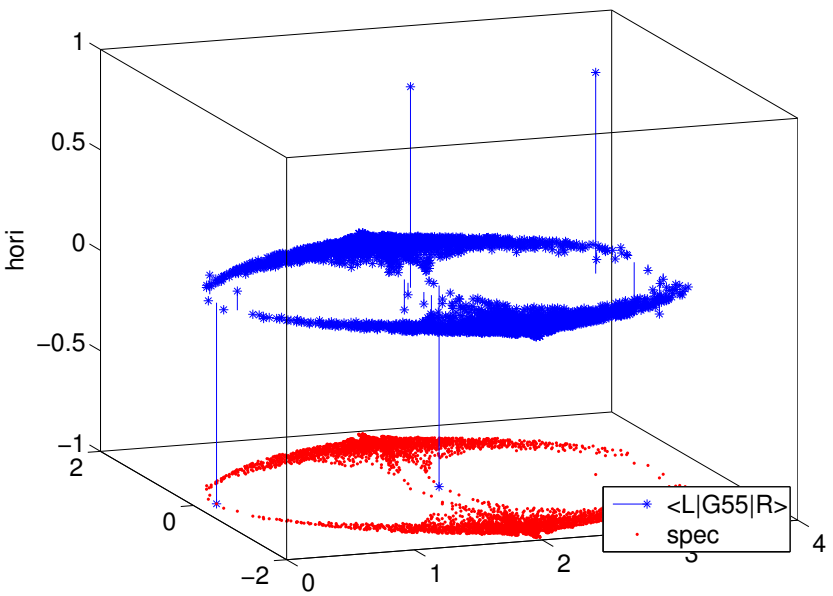

4D: $N C=3, \beta=5.8, L=6, T=6,|q|=1, c \_S W=1$

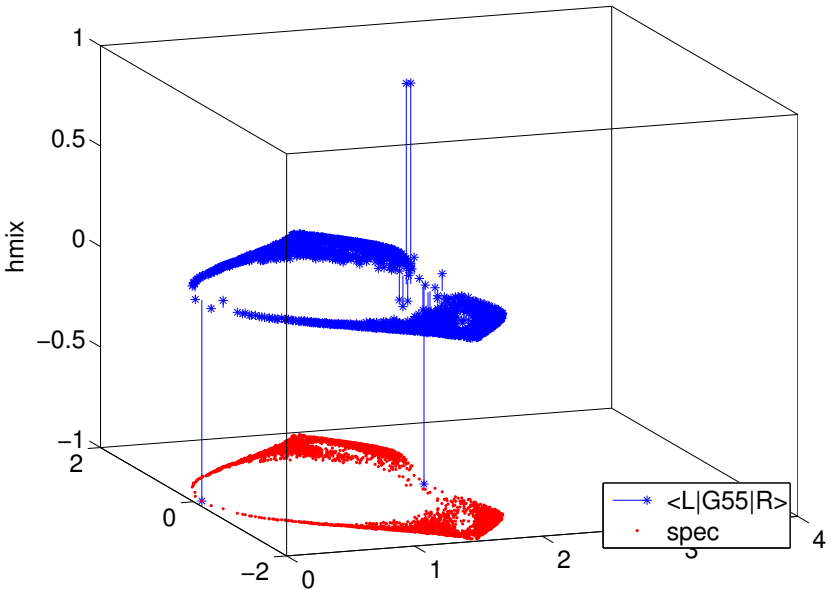

Figure 8: Needle plots of the chiralities with respect to $\Gamma_{55}$ of the improved operators $\left(c_{\mathrm{SW}}=1\right)$ above their eigenvalues. The LR-definition of $\left\langle.\left|\Gamma_{55}\right|.\right\rangle$ and three HEX smearings are used.

that the alternative operator $\sum_{\mu<\nu} \Xi_{\mu \nu} F_{\mu \nu}+$ h.c. does not lead to a similar improvement of the eigenvalues in the physical branch.

Fig. 6 displays the same kind of needle plots for the $\Gamma_{50}$ and $\Gamma_{55}$ chiralities of the eigenmodes of the operators (1) and (4) that were presented previously without improvement. Relative to Fig. 2 the most significant change is that the difference between $\langle L|| L$.$\rangle and \langle L|| R$.$\rangle in the$ physical branch is now less pronounced. This is a hint that the non-normality of the operator, when restricted to the physical subspace, is reduced by the improvement term.

Fig. 7 displays a feature that was not discussed in the unimproved case. The overlaps $\langle L|1| L\rangle$ and $\langle L|1| R\rangle$ are shown for the operators (1) and (4) - the former ones are trivially one, but the latter ones indicate how normal the operator is, and indeed this figure is close to 1 in the physical branch. In addition the taste chiralities are displayed; both $\left\langle L\left|\Gamma_{05}\right| L\right\rangle$ and $\left\langle L\left|\Gamma_{05}\right| R\right\rangle$ tend to assign each branch a fixed taste chirality which is not sensitive to topology of the background (as mentioned earlier, with our choices the taste chirality in the physical branch is $\left.\xi_{5}=-1\right)$. The conclusion is that the taste chiralities in the interacting case look similar to the free-field results presented in 12 . 
4D: $N C=3, \beta=5.8, L=6, T=6,|q|=1, c \_S W=1$

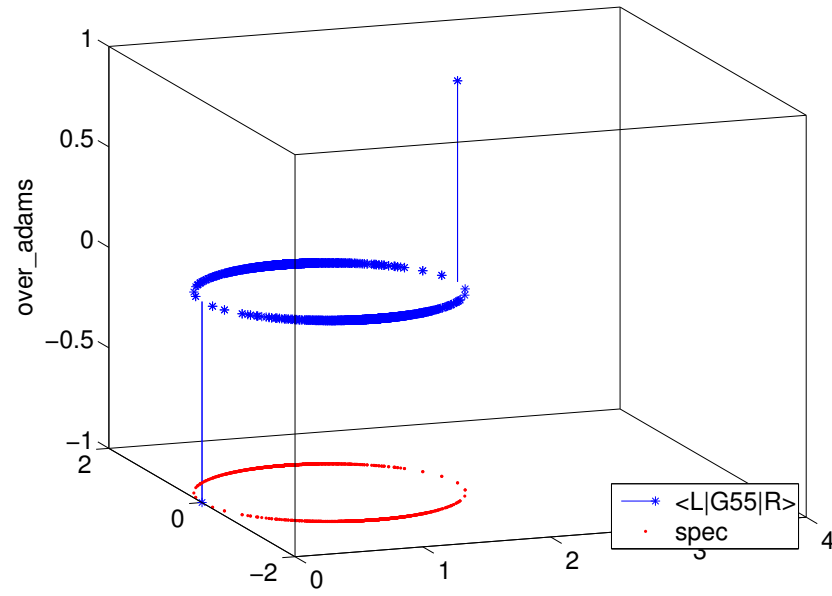

4D: $N C=3, \beta=5.8, L=6, T=6,|q|=1, c \_S W=1$

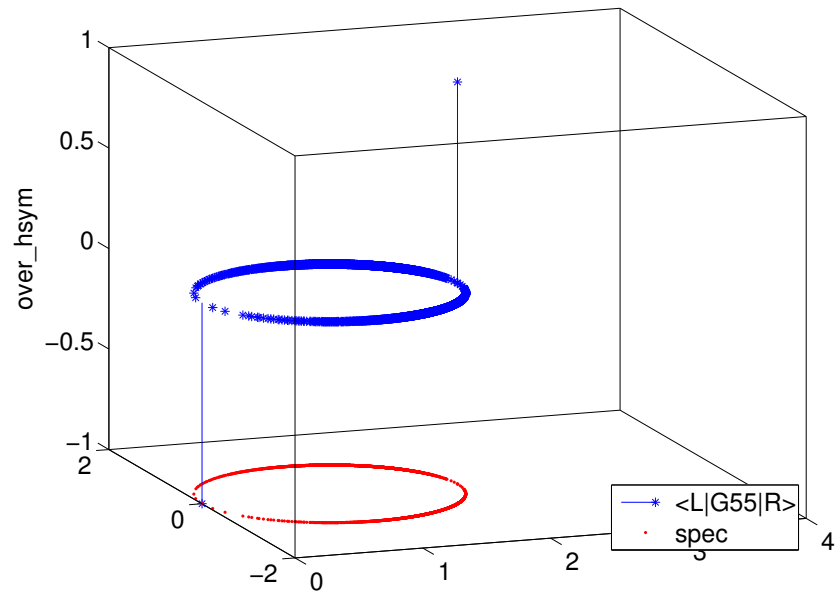

4D: $N C=3, \beta=5.8, L=6, T=6,|q|=1$, c_SW=1

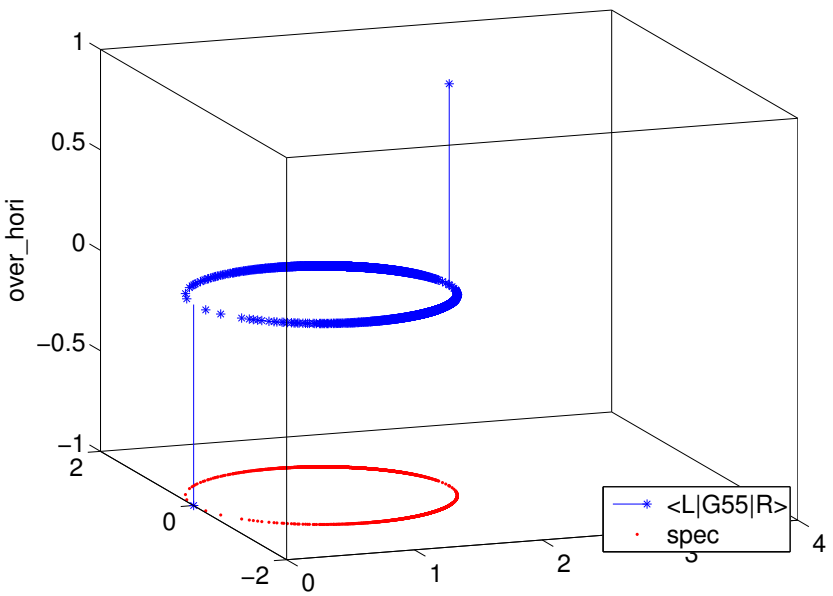

4D: $N C=3, \beta=5.8, L=6, T=6,|q|=1, c \_S W=1$

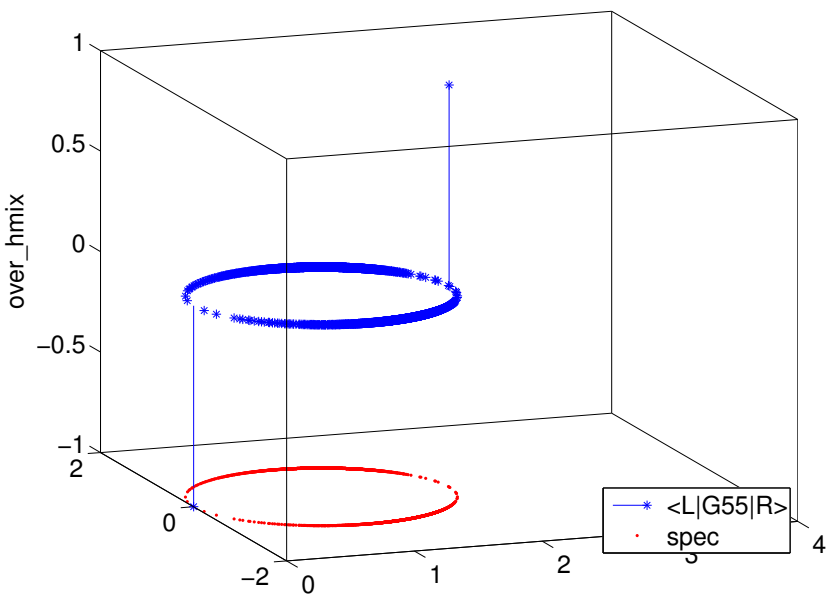

Figure 9: Needle plots of the chiralities with respect to $\Gamma_{55}$ of the overlap actions with improved kernels $\left(c_{\mathrm{SW}}=1\right)$. All $L / R$-definitions of $\left\langle.\left|\Gamma_{55}\right|.\right\rangle$ are equivalent; three HEX smearings are used.

Fig. 8 displays the $\left\langle L\left|\Gamma_{55}\right| R\right\rangle$ chiralities of all four operators (1) 4 ) with the clover term (25). These chiralities are very well pronounced (i.e. very close to \pm 1 ) and linked to the topology of the gauge background; a cut at \pm 0.5 is well suited to identify the chiral modes.

\section{Results with overlap projection}

The definition of the massless overlap Dirac operator takes the form [4,5]

$$
D_{\mathrm{ov}}=\frac{\rho}{a}\left(1+X\left(X^{\dagger} X\right)^{-1 / 2}\right)=\frac{\rho}{a}\left(1+\left(X X^{\dagger}\right)^{-1 / 2} X\right)
$$

where $X=a D_{\mathrm{ke}}-\rho$ and the kernel $D_{\mathrm{ke}}$ may be any undoubled fermion action [as is the case with [2 44] or a doubled one where the tastes in the physical branch share one chirality [as is the case with (1)]. Evidently, the overlap inherits the multiplicity of the kernel operator, e.g. two-fold with (1) as kernel. The canonical value of the shift parameter $0<\rho<2$ is $\rho=1$, a choice which we shall adopt in the following. Because the eigenvalue spectra are somewhat boring (the spectra lie on the shifted unit circle), we proceed directly to the chiralities. 
4D: $N C=3, \beta=5.8, L=6, T=6,|q|=1, c \_S W=1$

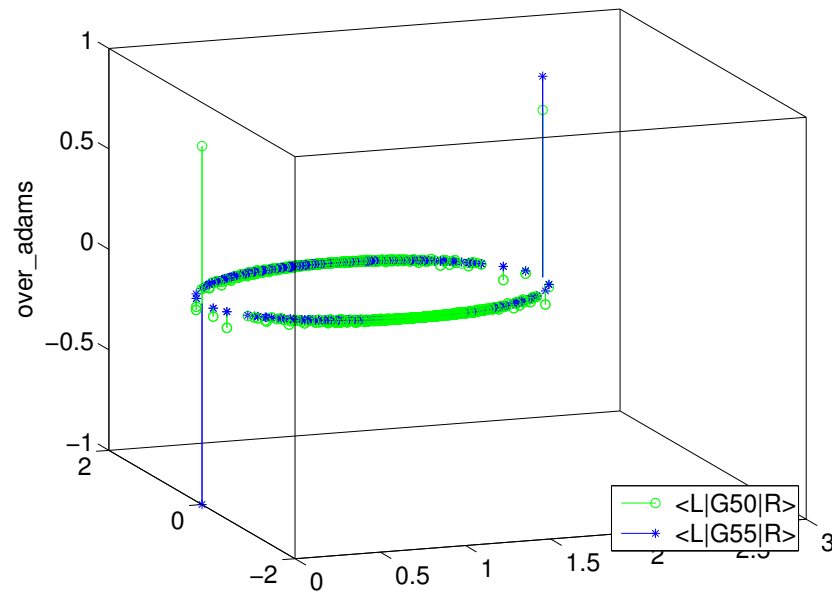

4D: $N C=3, \beta=5.8, L=6, T=6,|q|=1$, c_SW=1

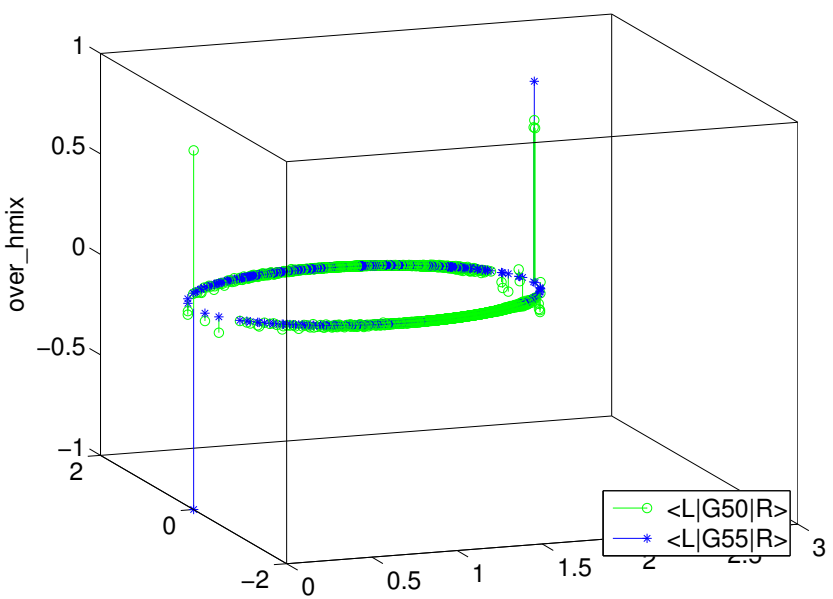

Figure 10: Needle plots of the chiralities of the overlap actions based on the Adams kernel (1) (left) or the mixed kernel (4) (right), with respect to $\Gamma_{50}$ (green circles) or $\Gamma_{55}=\epsilon$ (blue stars). Either kernel uses Symanzik improvement $\left(c_{\mathrm{SW}}=1\right)$ and three HEX smearings.

Fig. 9 displays the $\left\langle.\left|\Gamma_{55}\right|.\right\rangle$ chiralities of the overlap action built from the kernels (11 4). These operators are normal, i.e. the left-eigenvectors are daggered copies of the right-eigenvectors, viz. $\left\langle L_{i}|=| R_{i}\right\rangle^{\dagger}$, and the distinction between the four $\mathrm{L} / \mathrm{R}$-versions of $\left\langle.\left|\Gamma_{55}\right|.\right\rangle$ is now obsolete. The overlap procedure being a projection, together with the $\Gamma_{55}$ hermiticity of the kernel, ensures that the resulting $\left\langle.\left|\Gamma_{55}\right|.\right\rangle$ chiralities are exactly \pm 1 (topological modes) or exactly 0 (nontopological modes). As a side-effect in the conglomerate of unphysical branches (near $x=2$ ) only one type of $\Gamma_{55}$ chirality survives [contrary to what a naive shift of the corresponding modes of the kernel operator in Fig. 8 would suggest]. Thus the needle at $z=(x, y)=(2,0)$ is two-fold populated with the Adams kernel, but one-fold in all other cases.

Fig. 10 compares the $\left\langle.\left|\Gamma_{55}\right|.\right\rangle$ chiralities of the overlap operators with taste-split staggered kernel to the $\left\langle.\left|\Gamma_{50}\right|.\right\rangle$ chiralities (still on the same $|q|=1$ configuration). While the former are exactly \pm 1 (for the topological modes) the latter are not, in spite of the overlap projection. As discussed before this is a consequence of the kernels being $\Gamma_{55}$ hermitian but not $\Gamma_{50}$ hermitian.

As a practical point let us remark that the versions with unimproved kernels $\left(c_{\mathrm{SW}}=0\right)$ look superficially identical to the versions with clover improved kernels $\left(c_{\mathrm{SW}}=1\right)$ that were presented in the last two figures. Given that the combination of clover improvement and link smearing facilitated the separation between the physical branch and the unphysical branch(es), it is hardly surprising that the condition number in the overlap construction is significantly smaller in the latter case (which reduces the order of the polynomial/rational approximation to the sign function and thus the computational requirements). In short, it is strongly recommended to equip the kernel operator with a clover term and overall link smearing.

Fig. 11 contains two addenda to the overlap theme. In the left panel the $\left\langle.\left|\gamma_{5}\right|.\right\rangle$ chiralities of the overlap operator with Wilson kernel are shown, and the analogy to Fig.10 is evident. In the right panel the taste chiralities $\left\langle.\left|\Gamma_{05}\right|.\right\rangle$ of the overlap operator with Adams kernel are shown, and the smooth pattern that would show up in the kernel only in the $\langle L|| L$.$\rangle version$ (cf. Fig.7) is now generic (as the $\mathrm{L} / \mathrm{R}$ distinction is gone). 
4D: $N C=3, \beta=5.8, L=6, T=6,|q|=1$, c_SW=1

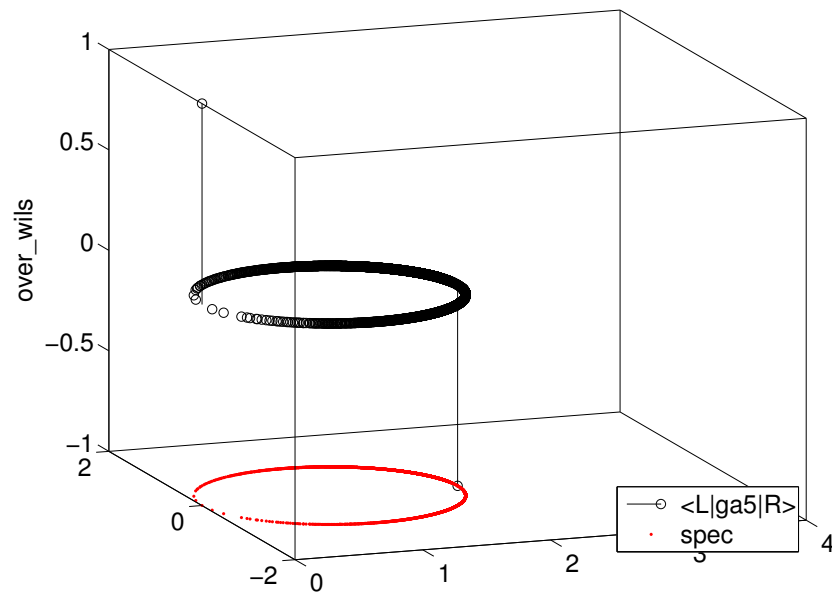

4D: $N C=3, \beta=5.8, L=6, T=6,|q|=1$, c_SW=1

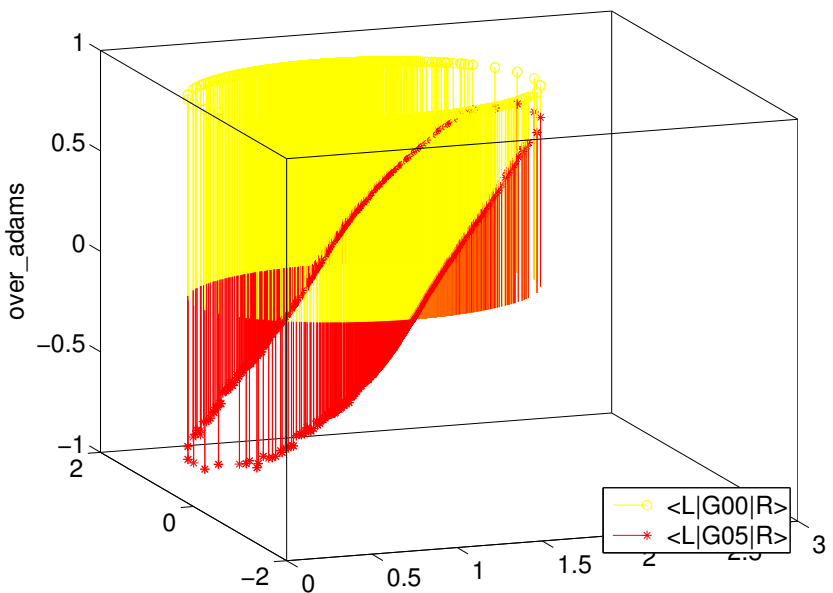

Figure 11: Needle plot of the $\left\langle.\left|\gamma_{5}\right|.\right\rangle$ chiralities of the eigenmodes of the overlap action with clover improved $\left(c_{\mathrm{SW}}=1\right)$ Wilson kernel (left) and $\left\langle L\left|\Gamma_{05}\right| R\right\rangle$ taste-chiralities of the overlap action with clover improved $\left(c_{\mathrm{SW}}=1\right)$ Adams kernel (right).

\section{Eigenvalue comparison to Wilson-type actions}

Since the taste-split staggered operators (1 4 -4 were found to behave, on many practical issues, like Wilson fermions, it seems instructive to compare their eigenvalue spectra (still on the same $|q|=1$ configuration) to those of the Wilson [1] and Brillouin [28] action.

Fig. 12 gives such a comparison, without improvement (left) and with tree-level clover improvement (right). Throughout gauge links $V_{\mu}(x)$ are used which have undergone 1 or 3 steps of HEX smearing [22]. The striking feature with $c_{\mathrm{SW}}=0$ is that the lowest non-topological modes of all operators sit essentially in the same place (with two nearly degenerate copies in case of the Adams operator), but the tendency of the topological modes to get "soaked into the belly" is different (the Adams operator fares best, the Wilson operator fares worst on this point). With $c_{\mathrm{SW}}=1$ the situation is just opposite; the "soak in" phenomenon seems cured with all operators, but now the position of the physical branch varies, and this time the Brillouin operator performs best. A peculiar issue with the latter action is the "thorn" of eigenvalues that grows from the doubler point 2 into the belly, once the clover term starts spreading the 15 unphysical modes. It is not clear whether this is an advantage (it might facilitate topology changes) or a disadvantage (topology might be less clearly defined) of this action - in the latter case it might be cured by multiplying its clover term with a factor $\left(1+\triangle^{\text {Bri }} / 4\right)$, with symmetrization, where $\triangle^{\mathrm{Bri}}$ is defined in [28]. In short these spectra underline the value of the improvement term (25) and suggest that - at least for some applications - the actions (1, 4) might fare well. The conceptual issue related to the rotational symmetry breaking of (4) is discussed in Sec.7.

The reader might wonder whether further elements of the set of recently tried modifications to the Wilson action (other smearings, twisted-mass term, etc.) may prove useful in the context of taste-split staggered actions. Regarding the link smearing it is clear that the fermion action is fairly insensitive to the details of this procedure. In view of dynamical fermion simulations the stout, n-HYP and HEX recipes are most interesting, since they can be combined with the (R)HMC algorithm, but the reader is free to select a different procedure. A twisted-mass term $\mathrm{i} \mu\left(\epsilon \otimes \tau_{3}\right)$ [based on the exact $\epsilon=\gamma_{5} \otimes \xi_{5}$ symmetry] would bring a lower bound on the determinant, 
4D: $N C=3, \beta=5.8, L=6, T=6,|q|=1, C \_S W=0$
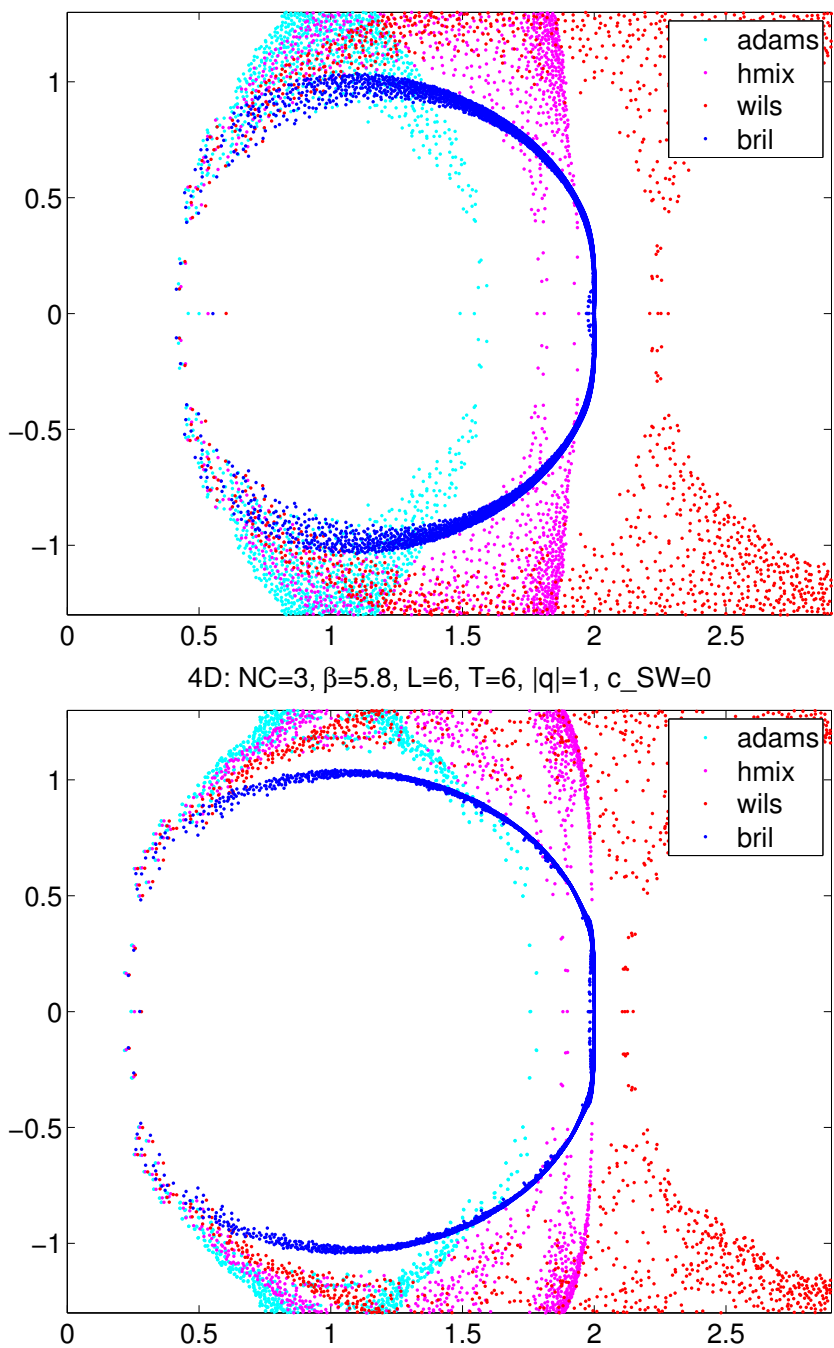

4D: $N C=3, \beta=5.8, L=6, T=6,|q|=1, C \_S W=1$
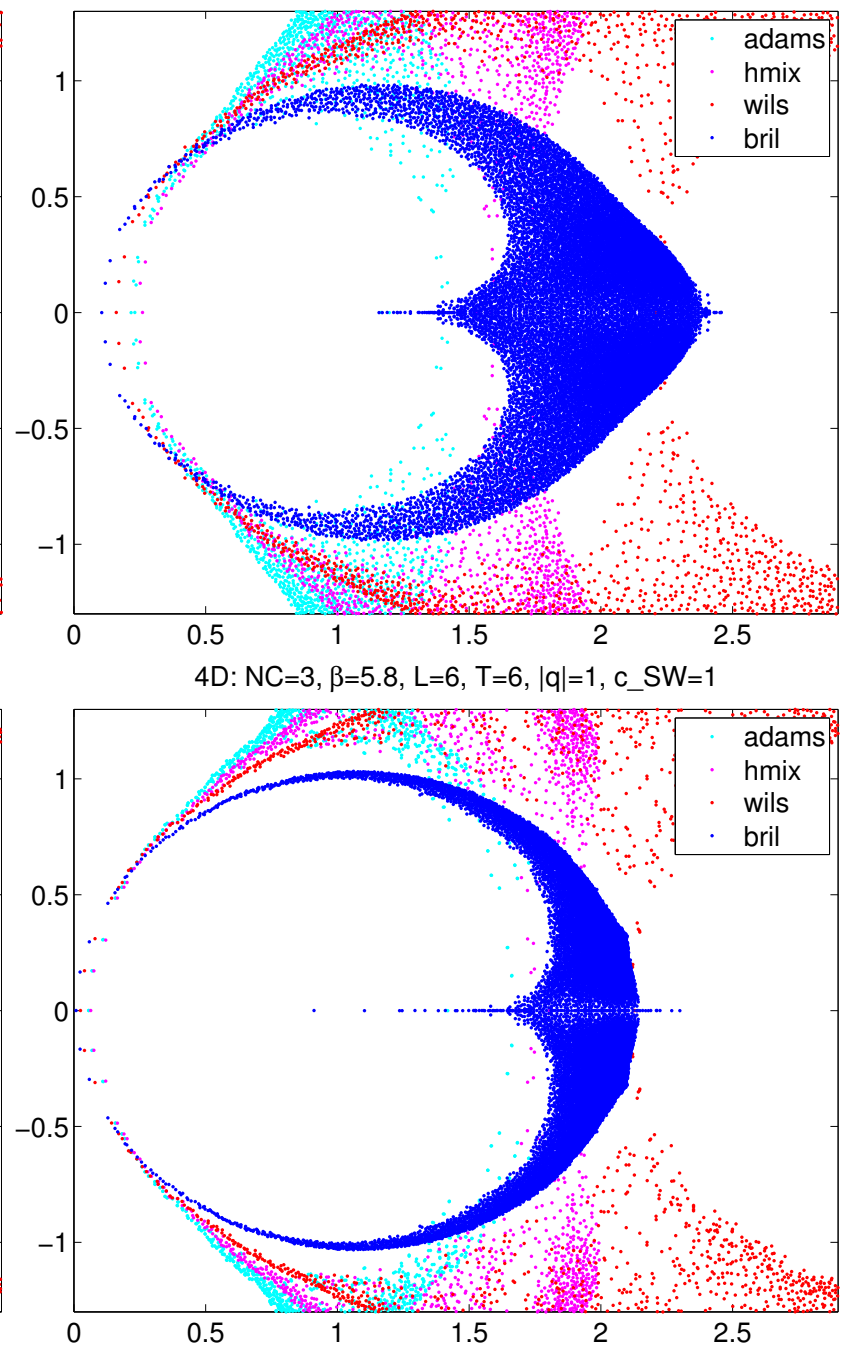

Figure 12: Eigenvalue spectra of the operators (1) and (4) and of the Wilson and Brillouin operators on a gauge configuration with topological charge $|q|=1$, with $c_{\mathrm{SW}}=0$ (left) and $c_{\mathrm{SW}}=1$ (right), after 1 (top) and 3 (bottom) steps of HEX smearing.

but it requires pairs of Hoelbling fermions. This would be interesting if it allows to cure the problem of rotational symmetry breaking discussed in Sec.7. A relevant issue is how the cut-off induced isospin breaking of such a formulation compares to the one in the Adams formulation (1). Clearly, such questions are well beyond the scope of this article.

\section{Signs of rotational symmetry breaking}

As mentioned in the introduction the taste-tensor mass term in the Hoelbling operators (2, 4 ) breaks the rotational [hypercubic] symmetry group $R_{\mu \nu}$ of the staggered action, leading to undesirable operators generated through quantum effects [11 14]. In consequence in a dynamical simulation with such an action one would expect to see signs of anisotropy, for instance unequal expectation values $\left\langle W_{r \times t}\right\rangle$ of planar Wilson loops oriented in the six $(\mu, \nu)$ planes [11]. 

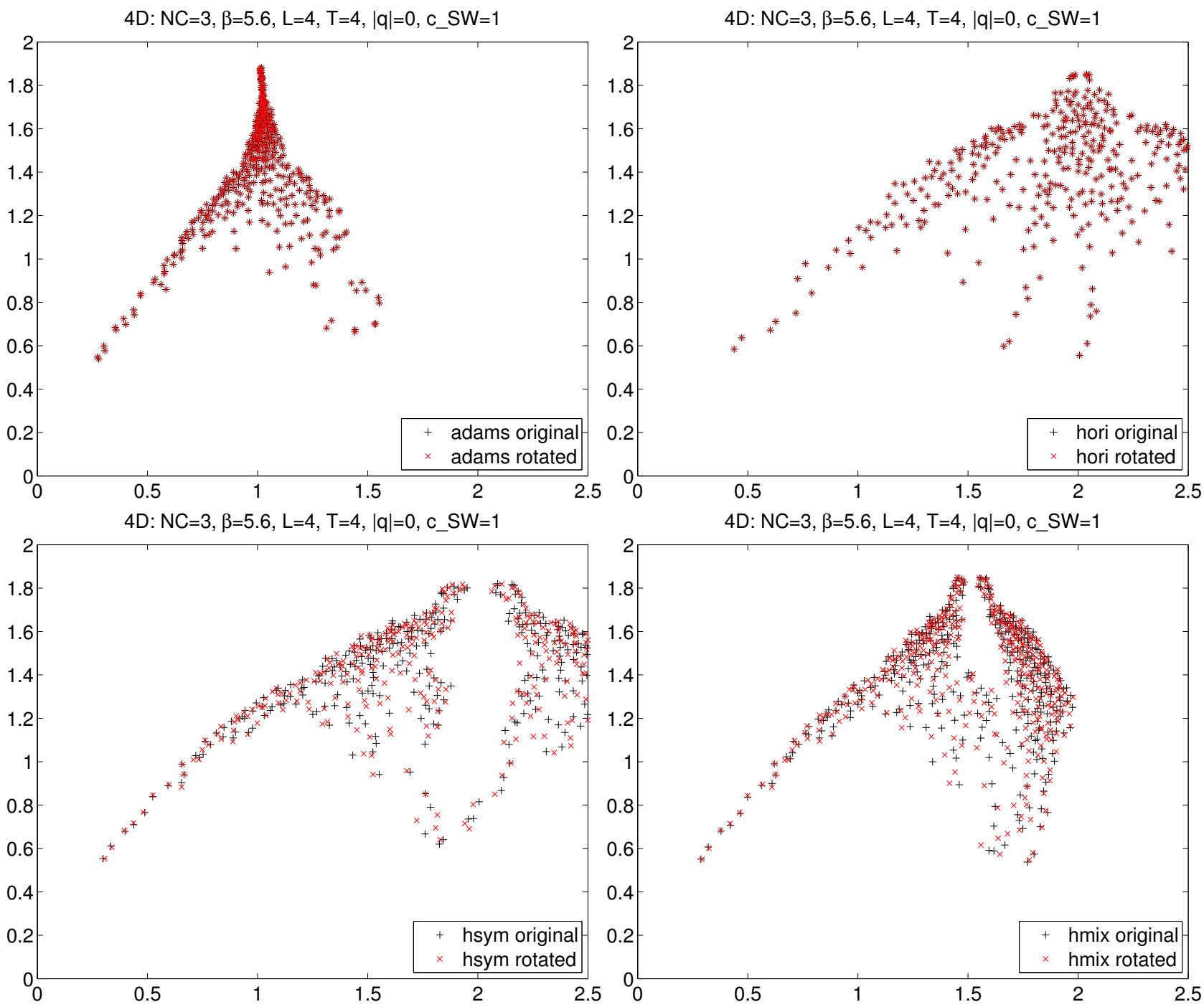

Figure 13: Eigenvalue spectra of the operators (1.2) (top) and (3.4) (bottom) with $c_{\mathrm{SW}}=1$, before and after rotating the gauge background in the $(1,2)$ plane.

Fig. 13 displays (half of) the eigenvalue spectra of the four operators (1, 4) on a single $4^{4}$ configuration before and after rotating the gauge background in the $(1,2)$ plane. The eigenvalue spectrum of the Adams operator (1) is unaffected, as it must be, since $R_{\mu \nu}$ is a symmetry. Also the eigenvalue spectrum of the original Hoelbling operator (2) with $M_{\text {Hori }}=\Xi_{12}$ is unaffected, but it is worth noting that the same statement does not hold true for other rotations [e.g. in the $(2,3)$ plane]. The eigenvalue spectrum of the symmetrized operator (3) is affected by the rotation in the $(1,2)$ plane, but interestingly the change is predominantly due to the modes in the first doubler branch near $\operatorname{Re}(z)=2$; the physical low-energy modes get barely changed. The same statement is found to hold true for the mixed operator (4). In either case it was checked that the determinant does not show an accidental symmetry, i.e. it changes too.

In short we arrive at the tantalizing situation where the symmetrized Hoelbling operator (3) shows "less symmetries" than the original variety (2). The breaking is predominantly due to the UV modes (which are susceptible to other changes, too, e.g. a switching between periodic 
and antiperiodic boundary conditions in one direction) and barely affects the IR properties of the operator. This raises a number of questions. Could it be that the breaking disappears in the limit of infinite statistics? If not, could it be that the breaking disappears on the way to the continuum? Likely the proper tool to answer such questions is an operator analysis in the Symanzik effective theory [11] and/or a perturbative analysis as pursued for minimally doubled actions 29], but a careful study is well beyond the scope of this article.

\section{Summary}

The main findings of this paper may be summarized as follows:

1. The discretizations (1) and (3), as proposed by Adams [9] and Hoelbling [10], respectively, and the new linear combination (4) yield staggered fermions with non-standard (i.e. taste non-singlet) mass terms (cf. Sec.2). They are "hybrids" in the sense that they distribute the spinor degrees of freedom over spacetime in the manner of staggered fermions, while technically being close to Wilson fermions, with additive mass renormalization and the same consequences of non-normality $(\langle L|$ and $|R\rangle$ eigenmodes $)$.

2. The main technical challenge of staggered fermions - the non-coincidence of the exact (tasteful) $\epsilon \equiv \Gamma_{55}$ and the non-exact (taste-singlet) $\Gamma_{50}$ chiral symmetries - persists. What changes is the details of how the operator is sensitive to the topological charge $q$ of the gauge background. With just a scalar mass term, $\left\langle V\left|\Gamma_{55}\right| V\right\rangle$ is zero between all eigenmodes of the operator and only $\left\langle V\left|\Gamma_{50}\right| V\right\rangle$ is sensitive to topology. With a sufficiently large taste-tensor or taste-pseudoscalar mass term, as is the case in (1) 4 ) with $r=1$, the matrix element $\left\langle L\left|\Gamma_{50}\right| R\right\rangle$ continues to be sensitive to topology (with equal sign in the physical and the unphysical branches), while $\left\langle L\left|\Gamma_{55}\right| R\right\rangle$ acquires an even better sensitivity to topology (with net zero sign across all branches).

3. The physical branches of the operators (1-4) show a cancellation-free sensitivity to topology with $2|q|$ or $|q|$ exactly real modes. But they also suffer from the same symptoms of chiral symmetry breaking with induced $O(a)$ cut-off effects as Wilson fermions, and it seems thus natural to try similar remedies. In this article it is conjectured that the leading Symanzik improvement term to these actions takes the form (25), and the connection to the overlap procedure suggests that the tree-level improvement coefficient is again $c_{\mathrm{SW}}=1$. The combination of Symanzik improvement and link-smearing is found to have a pronounced (and beneficial) effect on the formation of a "belly" between the physical and the unphysical branches in the eigenvalue plot (which, in turn, is seen as a sign that the mixing with other operators is suppressed). By explicitly rotating the gauge background it is confirmed that the taste-tensor mass term in (2,4) breaks the rotational symmetry group, but this seems to be linked to UV modes which barely affect IR physics.

4. The kernel operators (1) and (4), once equipped with link smearing and a clover term, bear the promise of a cheap overlap construction (in contrast to the mild savings that were found without these ingredients [12]) due to a moderate spectral range over which the sign function is to be constructed. Accordingly, a combination of these two overlap operators might be an attractive option for simulating QCD with $2+1+1$ active flavors. 
Acknowledgments: The author wishes to thank Christian Hoelbling and Stefan Krieg for useful discussion. This work was supported in part by the German SFB TRR-55.

\section{A Leading terms generated by the overlap procedure}

To facilitate the analysis of the necessary counterterms for $O(a)$ improvement of the taste-split staggered actions (1, 4) let us begin with a reflection on the situation with Wilson fermions. The idea that for Wilson fermions the overlap procedure of [4,5] would automatically generate the necessary improvement term is mentioned in the review by Niedermayer [30]. Unfortunately, this account is not very verbose, and this is why we attempt a short summary.

One essential ingredient in what follows is the continuum relation

$$
\not D^{2}=D_{\mu} \gamma_{\mu} D_{\nu} \gamma_{\nu}=\left(\frac{1}{2}\left\{\gamma_{\mu}, \gamma_{\nu}\right\}+\frac{1}{2}\left[\gamma_{\mu}, \gamma_{\nu}\right]\right) D_{\mu} D_{\nu}=D^{2}+\sum_{\mu<\nu} \sigma_{\mu \nu} F_{\mu \nu}
$$

with $\sigma_{\mu \nu}=\frac{\mathrm{i}}{2}\left[\gamma_{\mu}, \gamma_{\nu}\right]$ which is also dubbed $\gamma_{\mu \nu}$ in Sec.2. The other ingredient is the definition (26) of the overlap procedure with $X=a D_{\mathrm{ke}}-\rho$. This definition does not rely on any special property of the kernel operator $D_{\mathrm{ke}}$. With an argument which is $\gamma_{5}$ or $\epsilon$ hermitian the procedure can be recast in a form which involves the sign function; this will be relevant for numerical applications with (1, 4) as a kernel but not for the analytical considerations below.

With the Wilson operator as kernel one starts from the transcription (here we follow [31])

$$
D_{\mathrm{W}}=\sum_{\mu}\left\{\gamma_{\mu} \nabla_{\mu}-\frac{a}{2} \triangle_{\mu}\right\} \sim \not D-\frac{a}{2} D^{2}+O\left(a^{2}\right)
$$

in terms of continuum operators. Here $\nabla_{\mu}$ and $\triangle_{\mu}$ denote the gauge covariant first and second discrete derivative, respectively, the latter one to be distinguished from the continuum $D_{\mu}^{2}$. This implies $X=-\rho+a \not D-\frac{a^{2}}{2} D^{2}+O\left(a^{3}\right)$ and $X^{\dagger}=-\rho-a \not D-\frac{a^{2}}{2} D^{2}+O\left(a^{3}\right)$ and thus

$$
X^{\dagger} X=\rho^{2}-(1-\rho) a^{2} D^{2}-a^{2} \sum_{\mu<\nu} \sigma_{\mu \nu} F_{\mu \nu}+O\left(a^{3}\right)
$$

in the physical branch. Next we use the expansion $(1+z)^{-1 / 2}=1-\frac{1}{2} z+O\left(z^{2}\right)$ to arrive at

$$
\left(X^{\dagger} X\right)^{-1 / 2}=\frac{1}{\rho}\left(1+\frac{1-\rho}{2 \rho^{2}} a^{2} D^{2}+\frac{a^{2}}{2 \rho^{2}} \sum_{\mu<\nu} \sigma_{\mu \nu} F_{\mu \nu}+O\left(a^{3}\right)\right)
$$

and upon multiplying this with $X$ we find

$$
X\left(X^{\dagger} X\right)^{-1 / 2}=-1+\frac{a}{\rho} \not D-\frac{a^{2}}{2 \rho^{2}} D^{2}-\frac{a^{2}}{2 \rho^{2}} \sum_{\mu<\nu} \sigma_{\mu \nu} F_{\mu \nu}+O\left(a^{3}\right)
$$

with the consequence that the overlap operator relates to the continuum Dirac operator like

$$
D_{\mathrm{ov}}=\not D-\frac{a}{2 \rho} D^{2}-\frac{a}{2 \rho} \sum_{\mu<\nu} \sigma_{\mu \nu} F_{\mu \nu}+O\left(a^{2}\right)
$$

The second term may be removed by a field rotation, but the third term indicates that $O(a)$ improvement of $D_{\mathrm{W}}$ calls for a term $-\frac{c_{\mathrm{SW}}}{2} \sum_{\mu<\nu} \sigma_{\mu \nu} F_{\mu \nu}$ with $c_{\mathrm{SW}}=1$ at tree level. 
The task is now to pipe the taste-split staggered operators (1-4) through the overlap procedure and to see which counterterms are generated. First we need to transcribe the staggered operator in terms of continuum operators on the blocked lattice $(b=2 a)$. For (1) we find

$$
\begin{aligned}
D_{\mathrm{A}} & =\sum_{\mu}\left\{\left(\gamma_{\mu} \otimes 1\right) \nabla_{\mu}-\frac{b}{2}\left(\gamma_{5} \otimes \xi_{\mu}^{*} \xi_{5}\right) \triangle_{\mu}\right\}+r\left(1 \otimes \xi_{5}\right)+r(1 \otimes 1) \\
& \sim \not D-\frac{b}{2} \gamma_{5} D^{2}+O\left(b^{2}\right)
\end{aligned}
$$

where in the second line [which is supposed to capture the effect on the physical branch in terms of continuum operators] we use that the violation of Lorentz symmetry takes place exclusively in the taste space. Hence, the only relevant difference to $(28)$ is an additional factor of $\gamma_{5}$. This implies $Y=-\rho+b \not D-\frac{b^{2}}{2} \gamma_{5} D^{2}+O\left(b^{3}\right)$ and $Y^{\dagger}=-\rho-b \not D-\frac{b^{2}}{2} \gamma_{5} D^{2}+O\left(b^{3}\right)$ and thus

$$
Y^{\dagger} Y=\rho^{2}-\left(1-\rho \gamma_{5}\right) b^{2} D^{2}-b^{2} \sum_{\mu<\nu} \gamma_{\mu \nu} F_{\mu \nu}+O\left(b^{3}\right)
$$

in the physical branch. With the same expansion as before we obtain

$$
\left(Y^{\dagger} Y\right)^{-1 / 2}=\frac{1}{\rho}\left(1+\frac{1-\rho \gamma_{5}}{2 \rho^{2}} b^{2} D^{2}+\frac{b^{2}}{2 \rho^{2}} \sum_{\mu<\nu} \gamma_{\mu \nu} F_{\mu \nu}+O\left(b^{3}\right)\right)
$$

and upon multiplying this with $Y$ we find

$$
Y\left(Y^{\dagger} Y\right)^{-1 / 2}=-1+\frac{b}{\rho} \not D-\frac{b^{2}}{2 \rho^{2}} D^{2}-\frac{b^{2}}{2 \rho^{2}} \sum_{\mu<\nu} \gamma_{\mu \nu} F_{\mu \nu}+O\left(b^{3}\right)
$$

which agrees with the expression (31) which we found in the Wilson case. The bottom line is that in order to cancel the $O(b)$ effects we need to add a term which acts on the physical branch like $-\frac{c_{\mathrm{SW}}}{2} \sum_{\mu<\nu} \gamma_{\mu \nu} F_{\mu \nu}$, with $c_{\mathrm{SW}}=1$ at tree level. To improve the actual action (1) the obvious replacement is $\gamma_{\mu \nu} \rightarrow \Gamma_{\mu \nu}$. Finally, to ensure gauge covariance and hermiticity this expression needs to be symmetrized in the manner of (25).

None of the manipulations listed above did refer to the details of the taste lifting term $\propto r$ in (1 4 ); in fact an essential ingredient was that this term is effectively 0 in the physical branch. It follows that the operator (25) removes the leading cut-off effects in all taste-split staggered actions, with $c_{\mathrm{SW}}=1$ at tree-level, regardless of the multiplicity of the physical branch. 


\section{References}

[1] K.G. Wilson, Phys. Rev. D 10, 2445 (1974).

[2] L. Susskind, Phys. Rev. D 16, 3031 (1977).

[3] A. Bazavov, D. Toussaint, C. Bernard, J. Laiho, C. DeTar, L. Levkova, M. B. Oktay and S. Gottlieb et al., Rev. Mod. Phys. 82, 1349 (2010) arXiv:0903.3598.

[4] H. Neuberger, Phys. Lett. B 417, 141 (1998) hep-lat/9707022.

[5] H. Neuberger, Phys. Lett. B 427, 353 (1998) [hep-lat/9801031].

[6] H. S. Sharatchandra, H. J. Thun and P. Weisz, Nucl. Phys. B 192, 205 (1981).

[7] H. Kluberg-Stern, A. Morel, O. Napoly and B. Petersson, Nucl. Phys. B 220, 447 (1983).

[8] M. F. L. Golterman and J. Smit, Nucl. Phys. B 245, 61 (1984).

[9] D. H. Adams, Phys. Lett. B 699, 394 (2011), arXiv:1008.2833.

[10] C. Hoelbling, Phys. Lett. B 696, 422 (2011), arXiv:1009.5362.

[11] Compare the talk given by S. Sharpe at the Kyoto workshop in 02/2012:

http://www.phys.washington.edu/ ${ }^{\sim}$ sharpe/sharpe_kyoto12.pdf

[12] P. de Forcrand, A. Kurkela and M. Panero, JHEP 1204, 142 (2012) arXiv:1202.1867].

[13] T. Misumi, T. Z. Nakano, T. Kimura and A. Ohnishi, Phys. Rev. D 86, 034501 (2012) arXiv:1205.6545.

[14] T. Misumi, PoS LATTICE 2012, 005 (2012) arXiv:1211.6999].

[15] M. Creutz, T. Kimura and T. Misumi, JHEP 1012, 041 (2010) arXiv:1011.0761.

[16] M. Creutz, T. Kimura and T. Misumi, Phys. Rev. D 83, 094506 (2011) arXiv:1101.4239.

[17] T. Kimura, S. Komatsu, T. Misumi, T. Noumi, S. Torii and S. Aoki, JHEP 1201, 048 (2012) arXiv:1111.0402.

[18] L. H. Karsten and J. Smit, Nucl. Phys. B 183, 103 (1981).

[19] J. Smit and J. C. Vink, Nucl. Phys. B 286, 485 (1987).

[20] S. J. Hands and M. Teper, Nucl. Phys. B 347, 819 (1990).

[21] G. C. Donald, C. T. H. Davies, E. Follana and A. S. Kronfeld, Phys. Rev. D 84, 054504 (2011) arXiv:1106.2412.

[22] S. Capitani, S. Durr and C. Hoelbling, JHEP 0611, 028 (2006) hep-lat/0607006.

[23] I. Hip, T. Lippert, H. Neff, K. Schilling and W. Schroers, Phys. Rev. D 65, 014506 (2001) hep-lat/0105001.

[24] K. Symanzik, Nucl. Phys. B 226, 187 (1983).

[25] B. Sheikholeslami and R. Wohlert, Nucl. Phys. B 259, 572 (1985).

[26] M. Luscher, S. Sint, R. Sommer and P. Weisz, Nucl. Phys. B 478, 365 (1996) heplat/9605038.

[27] M. Luscher, S. Sint, R. Sommer, P. Weisz and U. Wolff, Nucl. Phys. B 491, 323 (1997) hep-lat/9609035.

[28] S. Durr and G. Koutsou, Phys. Rev. D 83, 114512 (2011) arXiv:1012.3615.

[29] S. Capitani, M. Creutz, J. Weber and H. Wittig, JHEP 1009, 027 (2010) arXiv:1006.2009].

[30] F. Niedermayer, Nucl. Phys. Proc. Suppl. 73, 105 (1999) hep-lat/9810026.

[31] H. Ikeda and S. Hashimoto, PoS LAT 2009, 082 (2009) arXiv:0912.4119. 